\title{
MiKLÒs HORVÁTH \\ Local uniform convergence of the Riesz means of Laplace and Dirac expansions
}

Annales de la faculté des sciences de Toulouse $6^{e}$ série, tome $6, \mathrm{n}^{\circ} 4$ (1997), p. 653-696

<http://www.numdam.org/item?id=AFST_1997_6_6_4_653_0>

(C) Université Paul Sabatier, 1997, tous droits réservés.

L'accès aux archives de la revue «Annales de la faculté des sciences de Toulouse » (http://picard.ups-tlse.fr/ annales/) implique l'accord avec les conditions générales d'utilisation (http://www.numdam.org/conditions). Toute utilisation commerciale ou impression systématique est constitutive d'une infraction pénale. Toute copie ou impression de ce fichier doit contenir la présente mention de copyright.

\section{Numdam}

Article numérisé dans le cadre du programme

Numérisation de documents anciens mathématiques

http://www.numdam.org/ 


\title{
Local uniform convergence of the Riesz means of Laplace and Dirac expansions ${ }^{(*)}$
}

\author{
MiKLòs HoRVÁTH ${ }^{(1)}$
}

\begin{abstract}
RÉSUMÉ. - On considère des extensions arbitraires non autoadjointes à spectre discret de l'opérateur de Laplace défini sur un domaine borné $\Omega$. On étudie les développements spectraux associés. On démontre que si la fonction développée se trouve dans un espace convenable et s'annule près du bord, alors le développement converge uniformément dans chaque compact $K \subset \Omega$. On démontre aussi un principe de localisation. Finalement, on obtient les mêmes résultats pour les développements suivant les fonctions propres de l'opérateur de Dirac.

Abstract. - We consider arbitrary nonselfadjoint extensions of the Laplace operator with discrete spectrum, defined in a domain $\Omega$. We study the corresponding spectral expansions. We prove that if the expanded function is sufficiently smooth and vanishes near the boundary then the expansion converges locally uniformly. We prove also a localization principle. In the final part of the paper, we obtain the same results for the Dirac expansions.
\end{abstract}

\section{Introduction}

This paper investigates nonselfadjoint Laplace operators with arbitrary complex discrete spectrum. If the eigenfunctions form a Riesz basis in $L_{2}$ (see [26]), it makes possible to study the expansions in this basis. We prove two theorems. The first one states that if $f$ is sufficiently smooth then the Riesz summation of the expansion of $f$ converge to $f$ locally uniformly. Theorem 2 states a localization principle like that of Riemann

(*) Reçu le 11 janvier 1995

(1) Technical University of Budapest, Institute of Mathematics, Department of Analysis, H-1111 Budapest Stoczek u. 2 (Hongrie)

e-mail : horvath@math.bme.hu

Supported by the Hungarian National Scientific Research Fund OTKA T 014244. 


\section{Horváth}

for ordinary Fourier series. The class $H_{p}^{\alpha}$ of smooth functions are defined by finite differences. Since $H_{p}^{\alpha}$ contains most of the known function classes (Sobolev classes, Liouville, Zygmund-Hölder, Besov classes; [21] or [4], [24]), hence our theorems hold also for those classes (with the same indices $\alpha$ and $p$ ). To avoid technical difficulties in the boundary of the domain $\Omega$ considered, we assume everywhere that the function expanded vanishes near the boundary. As we see from the statement of theorems, if we increase the summation order $s$, the convergence holds also for less smooth functions. The convergence is proved by the usual Banach-Steinhaus principle: the operator of taking Riesz means is bounded in norm (Lemma 2) and there is a dense subset where convergence holds (Lemma 5). The boundedness of the Riesz means is proved by the square sum estimate of Fourier coefficients (Lemma 4) and by the square sum estimate of eigenfunctions (15). Using these results, any sum $\sum c_{n}\left\langle f, v_{n}\right\rangle u_{n}(x)$ can be estimated by the Cauchy-Schwarz inequality; this is the basic idea in proving Lemma 2. The eigenfunctions $u_{n}$ satisfy the mean value formula (7) in which Bessel functions arise. Hence almost every proof below requires deep investigation of the properties of Bessel functions; that's why the paper became so lengthy.

In the second part of the paper, we extend Theorems 1 and 2 for Dirac expansions. We consider only the Dirac operator in empty space (i.e., with no vector and scalar potential). In this case the four coordinates of any eigenfunction of the Dirac operator are eigenfunctions of the Laplace operators with another eigenvalue, see (42). Hence the mean value formula (7) remains true and the whole proof of the Laplace case can be repeated.

\section{Setting of the problem}

In this paper we consider a bounded domain

$$
\Omega \subset \mathbb{R}^{N}, \quad N>1 .
$$

and a system $\left\{u_{n} \mid n \geq 1\right\}$ of eigenfunctions of the Laplace operator

$$
-\Delta u_{n}=\lambda_{n} u_{n} .
$$

We assume that the $u_{n}$ form a Riesz basis in $L_{2}(\Omega)$ (e.g. [26]) and denote $v_{n}$ its biorthogonal system defined by

$$
\begin{gathered}
\left\langle u_{n}, v_{k}\right\rangle=\delta_{n, k} . \\
-654-
\end{gathered}
$$


We don't assume that the $v_{n}$ are eigenfunctions. Let $\mu_{n}$ be a square root of $\lambda_{n}$ with $\Re \mu_{n} \geq 0$ and introduce the notations

$$
\rho_{n}:=\Re \mu_{n} \geq 0, \quad \nu_{n}:=\Im \mu_{n} .
$$

The Nikolskii classes $H_{p}^{\alpha}\left(\mathbb{R}^{N}\right)$ are defined by the following way. Let $\alpha>0$, $1 \leq p \leq \infty$ and let $k, \ell$ be nonnegative integers with

$$
k \geq \alpha-\ell>0 .
$$

Then the function $f$ belongs to $H_{p}^{\alpha}\left(\mathbb{R}^{N}\right)$ if and only if it has derivatives $f^{(\beta)},|\beta| \leq \ell$ in the distributional sense, $f \in L_{p}\left(\mathbb{R}^{N}\right)$ and

$$
M:=\sup _{\substack{h \in \mathbb{R}^{N} \\|\beta| \leq \ell}}\left(\left\|\Delta_{h}^{k} f^{(\beta)}(x)\right\|_{L_{p}}|h|^{\ell-\alpha}\right)<\infty .
$$

In this case

$$
\|f\|_{H_{p}^{\alpha}}:=\|f\|_{L_{p}}+M
$$

Here

$$
\Delta_{h}^{k} f(x)=\sum_{j=0}^{k}(-1)^{k-j}\left(\begin{array}{c}
k \\
j
\end{array}\right) f(x+j h)
$$

denotes the $k$-th difference of $f(x)$. It is known [24, $\S 2.3 .2]$ that $C_{0}^{\infty}\left(\mathbb{R}^{N}\right)$ is not dense in $H_{p}^{\alpha}\left(\mathbb{R}^{N}\right)$, so we introduce the notation

$$
\stackrel{\circ}{H}_{p}^{\alpha}(\Omega)
$$

as the closure of $C_{0}^{\infty}(\Omega)$ in $H_{p}^{\alpha}\left(\mathbb{R}^{N}\right)$. Introduce further the spectral expansion of a function $f \in L_{2}(\Omega)$ as

$$
f(x) \sim \sum\left\langle f, v_{n}\right\rangle u_{n}(x)
$$

its $\lambda$-th partial sum (resp. $\lambda$-th Riesz summation of order $s \geq 0$ ) is defined by

$$
\begin{aligned}
& \sigma_{\lambda}(f, x)=\sum_{\rho_{n}<\lambda}\left\langle f, v_{n}\right\rangle u_{n}(x) \\
& \sigma_{\lambda}^{s}(f, x)=\sum_{\rho_{n}<\lambda}\left\langle f, v_{n}\right\rangle u_{n}(x)\left(1-\rho_{n}^{2} \lambda^{-2}\right)^{s} .
\end{aligned}
$$

We aim to prove the following two statements. 
ThEOREM 1. - Let $f \in \stackrel{\circ}{H}_{p}^{\alpha}(\Omega), \operatorname{supp} f \subset \Omega, \alpha p>N, 1<p<\infty$, $0 \leq s<1 / 2$ and $\alpha+s \geq(N-1) / 2$. Then

$$
\sigma_{\lambda}^{s}(f, x) \stackrel{\lambda \rightarrow \infty}{\longrightarrow} f(x)
$$

locally uniformly in $x \in \Omega$.

THEOREM 2. Let $f \in \stackrel{\circ}{H}_{2}^{\alpha}(\Omega), \operatorname{supp} f \subset \Omega, \alpha>0,0 \leq s<1 / 2$ and $\alpha+s \geq(N-1) / 2$. Then

$$
\sigma_{\lambda}^{s}(f, x) \stackrel{\lambda \rightarrow \infty}{\longrightarrow} 0, \quad x \in \Omega \backslash \operatorname{supp} f
$$

locally uniformly in $\Omega \backslash \operatorname{supp} f$.

Remarks

1) For the case $\lambda_{n} \geq 0$ the above theorems were obtained by Il'in and Alimov [11]. In case $s=0$ these results were proved in Horváth ([6], [9]) for Liouville classes.

2) In case $N=1$ better results are known than Theorems 1 and 2 because we can transform them to the ordinary Fourier series by the equiconvergence theorem of Joó and Komornik [18]; see Zygmund [27].

3) Concerning the complex point spectrum, we mention the papers [19] of Komornik, [16], [15], [17] of Joó and [10] of Horváth, Joó and Komornik. The Riesz summation for selfadjoint Schrödinger operator is studied e.g. in Alimov, Joó [2] and Joó [12]-[14].

\section{Reduction of Theorems 1,2 to Lemma 1}

In this part of the paper the theorems are reduced to the following result.

Lemma 1 - Let $0<\alpha, 0 \leq s<1 / 2, \alpha+s=(N-1) / 2, f \in \stackrel{\circ}{H}_{2}^{\alpha}(\Omega)$, $\operatorname{supp} f \subset \Omega, x_{0} \in \Omega$ be a fixed point and suppose

$$
\begin{gathered}
f(x)=0 \text { for a.e. } x,\left|x-x_{0}\right|<\rho \\
-656-
\end{gathered}
$$


Local uniform convergence of the Riesz means of Laplace and Dirac expansions

for some $\rho>0$. Then for any sufficiently small $\varepsilon>0$ (namely if $2 \varepsilon<1 / 2-s$ ) the following estimates hold. If $x \in K \subset \Omega, K$ is closed, then

$$
\left|\sigma_{\lambda}^{s}(f, x)\right| \leq \begin{cases}c \lambda^{N / 2-\alpha}\|f\|_{H_{2}^{\alpha}} & \text { in case } \lambda \rho \leq 4 \pi, x \in K \\ c \rho^{\alpha-N / 2-\varepsilon}\|f\|_{H_{2}^{\alpha}} & \text { in case } \lambda \rho \geq 4 \pi, x \in K .\end{cases}
$$

Here the constants $c=c(K)$ may depend on $\varepsilon, \alpha, N, s$ but not on $x, \lambda$, $\rho, f$.

Supposing Lemma 1 proved, we can prove Theorems 1 and 2 as follows.

Lemma 2 - Let $\alpha>0,0 \leq s<1 / 2,1<p<\infty, 0<\alpha-N / p<1$, $\alpha+s=(N-1) / 2, f \in \stackrel{\circ}{H}_{p}^{\alpha}(\Omega)$, supp $f \subset \Omega$. Let further $K \subset \Omega$ be closed, then

$$
\left|\sigma_{\lambda}^{s}\left(f, x_{0}\right)\right| \leq c(K)\|f\|_{H_{p}^{\alpha}}, \quad x_{0} \in K
$$

The proof is almost identical to that of Lemma 4.3 of [2], so we omit the details.

Lemma $3 .-$ Let $0 \leq s<(N-1) / 2, \alpha p>N, \alpha+s \geq(N-1) / 2$, $1<p<\infty$. Then there exist $\beta$, q such that $0<\beta-N / q<1$, $\beta+s=(N-1) / 2,1<q<\infty$ and

$$
\stackrel{\circ}{H}_{p}^{\alpha}(\Omega) \subset \stackrel{\circ}{H}_{q}^{\beta}(\Omega) .
$$

Proof. - When $\alpha$ is diminishing, $H_{p}^{\alpha}\left(\mathbb{R}^{N}\right)$ and then $\stackrel{\circ}{H}_{p}^{\alpha}(\Omega)$ is growing. The lower bounds are $\alpha>N / p, \alpha \geq(N-1) / 2-s$. There are three possibilities after diminishing $\alpha$ as much as possible. If $\alpha+s=(N-1) / 2$, $0<\alpha-N / p<1$ then we are ready. If $\alpha+s=(N-1) / 2$ and $\alpha-N / p \geq 1$, we can diminish the value $p$; since $\Omega$ is bounded, $\stackrel{\circ}{H}_{p}^{\alpha}(\Omega)$ is growing. For $p=2, \alpha-N / p<0$, hence we find $p>2$ for which $0<\alpha-N / p<1$. Finally in case $\alpha+s>(N-1) / 2$ and $0<\alpha-N / p<1$ we can suppose also $N / p+s \geq(N-1) / 2$; we can apply then the imbedding theorem [24, $\S 2.8 .1]$

$$
H_{p}^{\alpha}\left(\mathbb{R}^{N}\right) \subset H_{q}^{\beta}\left(\mathbb{R}^{N}\right), \quad 1<p \leq q<\infty, \beta=\alpha-N\left(\frac{1}{p}-\frac{1}{q}\right)>0
$$


which implies

$$
\stackrel{\circ}{H}_{p}^{\alpha}(\Omega) \subset \stackrel{\circ}{H}_{q}^{\beta}(\Omega)
$$

Choose $q$ such that $(N-1) / 2-s-\alpha+N / p=N / q$. Then $1 / q<1 / p$ and if $\alpha$ is sufficiently close to $N / p$, then $0<1 / q$ hence $p<q<\infty$ and $\beta=(N-1) / 2-s=\alpha-N(1 / p-1 / q)>0$, so the imbedding (6) holds; finally $\beta+s=(N-1) / 2$ and $0<\beta-N / q=\alpha-N / p<1$. Lemma 3 is proved.

LemMa 4. - Let $\alpha, R>0, f \in H_{2}^{\alpha}\left(\mathbb{R}^{N}\right), \operatorname{supp} f \subset \Omega, \operatorname{dist}(\operatorname{supp} f, \partial \Omega)$ $\geq R$. Then

$$
\left(\sum_{\mu \leq \rho_{n} \leq 2 \mu}\left|\left\langle f, v_{n}\right\rangle\right|^{2}\right)^{1 / 2} \leq c \mu^{-\alpha}\|f\|_{H_{2}^{\alpha}}, \quad \mu \geq 1
$$

where $c=c(\alpha, R, N)$ is independent of $f$ and $\mu$.

Proof. - Choose a natural number $k$ such that

$$
2 k>\alpha>0
$$

and let

$$
0<h<\frac{R}{k}
$$

be arbitrary. Since $f \in L_{2}(\Omega)$, the series $f=\sum\left\langle f, v_{n}\right\rangle u_{n}(x)$ converges in $L_{2}(\Omega)$ and hence in $L_{1}(\Omega)$, too. Consequently, using the mean value formula

$$
\int_{\theta} u_{n}(x+r \theta) \mathrm{d} \theta=(2 \pi)^{N / 2} \frac{J_{N / 2-1}\left(\mu_{n} r\right)}{\left(\mu_{n} r\right)^{N / 2-1}} u_{n}(x)
$$


Local uniform convergence of the Riesz means of Laplace and Dirac expansions where $|\theta|=1$ runs over the unit sphere of $\mathbb{R}^{N}$, we see that

$$
\begin{aligned}
& \int_{|z| \leq h} f(x+z) \mathrm{d} z= \\
& \quad=\sum\left\langle f, v_{n}\right\rangle \int_{|z| \leq h} u_{n}(x+z) \mathrm{d} z \\
& \quad=\sum\left\langle f, v_{n}\right\rangle \int_{0}^{h} r^{N-1} \int_{\theta} u_{n}(x+r \theta) \mathrm{d} \theta \\
& =(2 \pi)^{N / 2} \sum\left\langle f, v_{n}\right\rangle u_{n}(x) \int_{0}^{h} r^{N-1} \frac{J_{N / 2-1}\left(\mu_{n} r\right)}{\left(\mu_{n} r\right)^{N / 2-1}} \mathrm{~d} r \\
& =(2 \pi)^{N / 2} \sum\left\langle f, v_{n}\right\rangle u_{n}(x)\left(\frac{h}{\mu_{n}}\right)^{N / 2} J_{N / 2}\left(\mu_{n} h\right) .
\end{aligned}
$$

Introduce the function

$$
\begin{aligned}
E(x, h):= & \sum_{j=0}^{k-1}(-1)^{j}\left(\begin{array}{c}
2 k \\
j
\end{array}\right) \frac{h^{-N}}{V_{N}} \int_{|u| \leq h} f(x+(k-j) u) \mathrm{d} u+ \\
& +\frac{(-1)^{k}}{2}\left(\begin{array}{c}
2 k \\
k
\end{array}\right) f(x)
\end{aligned}
$$

where $V_{N}=\pi^{N / 2} / \Gamma(N / 2+1)$ is the volume of the $N$-dimensional unit ball. We know that

$$
\begin{aligned}
& \Delta_{u}^{2 k} f(x-k u)= \\
& =\sum_{j=0}^{k-1}(-1)^{j}\left(\begin{array}{c}
2 k \\
j
\end{array}\right)(f(x+(k-j) u)+f(x-(k-j) u))+ \\
& \quad+(-1)^{k}\left(\begin{array}{c}
2 k \\
k
\end{array}\right) f(x) \\
& =\sum_{j=0}^{k-1}(-1)^{j}\left(\begin{array}{c}
2 k \\
j
\end{array}\right)(f(x+(k-j) u)+f(x-(k-j) u)-2 f(x)) .
\end{aligned}
$$

Consequently

$$
\begin{gathered}
E(x, h)=\frac{h^{-N}}{V_{N}} \int_{|u| \leq h} \frac{1}{2} \Delta_{u}^{2 k} f(x-k u) \mathrm{d} u . \\
-659-
\end{gathered}
$$




\section{Horváth}

Using the definition of $H_{2}^{\alpha}\left(\mathbb{R}^{N}\right)$ with $2 k>\alpha>0, \ell=0$, we get

$$
\begin{aligned}
\int_{\Omega}|E(x, h)|^{2} \mathrm{~d} x & =\frac{1}{4} \int_{\Omega}\left|\frac{h^{-N}}{V_{N}} \int_{|u|<h} \Delta_{u}^{2 k} f(x-k u) \mathrm{d} u\right|^{2} \mathrm{~d} x \leq \\
& \leq \frac{1}{4} \int_{\Omega} \frac{h^{-N}}{V_{N}} \int_{|u| \leq h}\left|\Delta_{u}^{2 k} f(x-k u)\right|^{2} \mathrm{~d} u \mathrm{~d} x \\
& \leq \frac{1}{4} \frac{h^{-N}}{V_{N}} \int_{|u| \leq h} \int_{\Omega}\left|\Delta_{u}^{2 k} f\right|^{2} \mathrm{~d} x \mathrm{~d} u \leq c\|f\|_{H_{2}^{\alpha} h^{2 \alpha}}^{2 \alpha}
\end{aligned}
$$

because $h k<R$. On the other hand, by (8) the coefficients of $E$ are

$$
\begin{aligned}
\left\langle E(\cdot, h), v_{n}\right\rangle= & \frac{(2 \pi)^{N / 2}}{V_{N}}\left\langle f, v_{n}\right\rangle\left(\sum_{j=0}^{k-1}(-1)^{j}\left(\begin{array}{c}
2 k \\
j
\end{array}\right) \frac{J_{N / 2}\left(\mu_{n} h(k-j)\right)}{\left(\mu_{n} h(k-j)\right)^{N / 2}}+\right. \\
& \left.+\frac{V_{N}}{(2 \pi)^{N / 2}} \frac{(-1)^{k}}{2}\left(\begin{array}{c}
2 k \\
k
\end{array}\right)\right) \\
= & \frac{(2 \pi)^{N / 2}}{V_{N}}\left\langle f, v_{n}\right\rangle F\left(\mu_{n} h\right)
\end{aligned}
$$

if the function $F(z)$ is defined by

$$
F(z)=V_{N}(2 \pi)^{-N / 2} \frac{(-1)^{k}}{2}\left(\begin{array}{c}
2 k \\
k
\end{array}\right)+\sum_{j=0}^{k-1}(-1)^{j}\left(\begin{array}{c}
2 k \\
j
\end{array}\right) \frac{J_{N / 2}((k-j) z)}{((k-j) z)^{N / 2}} .
$$

Since $\left\{u_{n}\right\}$ is a Riesz basis in $L_{2}(\Omega)$, we have (e.g. [26])

$$
\int_{\Omega}|E(x, h)|^{2} \mathrm{~d} x \geq c \sum\left|\left\langle E(\cdot, h), v_{n}\right\rangle\right|^{2}=c \sum\left|\left\langle f, v_{n}\right\rangle F\left(\mu_{n} h\right)\right|^{2} .
$$

Now, we prove that there exist constants $0<c_{1}<c_{2}<\infty$, depending only on $N$ and $k$ such that

$$
\inf _{c_{1} \leq \Re z \leq c_{2}}|F(z)|>0 .
$$

Taking into account (9) and (10) it implies the statement of Lemma 4 if we set $h=c / \mu$. To prove (11), we recall the expansion

$$
\begin{aligned}
J_{N / 2}(z) z^{-N / 2}=2^{-N / 2} & \sum_{n=0}^{\infty} \frac{(-1)^{n}}{2^{2 n} n ! \Gamma(N / 2+n+1)} z^{2 n} . \\
& -660-
\end{aligned}
$$


Local uniform convergence of the Riesz means of Laplace and Dirac expansions

It gives the following expansion for $F$ if we observe that the starting coefficient in (12) is $V_{N}(2 \pi)^{-N / 2}$ :

$$
\begin{aligned}
F(z) & =2^{-N / 2} \sum_{n=1}^{\infty} \frac{(-1)^{n} a_{n}}{2^{2 n} n ! \Gamma(N / 2+n+1)} z^{2 n} . \\
a_{n} & :=\sum_{j=0}^{k-1}(-1)^{j}\left(\begin{array}{c}
2 k \\
j
\end{array}\right)(k-j)^{2 n}=\frac{1}{2} \Delta_{1}^{2 k} g_{n}(x-k), \\
g_{n} & =x^{2 n}, \quad x=0 .
\end{aligned}
$$

It is known that

$$
\Delta_{1}^{2 k} g(x-k)=g^{(2 k)}(\varepsilon) \quad \text { for some }|\varepsilon|<k
$$

hence

$$
\left\{\begin{array}{l}
a_{1}=\cdots=a_{k-1}=0, \quad a_{k}=(2 k) ! \\
0 \leq a_{n} \leq 2^{2 k} k^{2 n}, \quad n>k
\end{array}\right.
$$

The entire function $F(z)$ has $2 k$-fold zero at $z=0$ and there exists $\delta>0$, $\delta=\delta(N, k)$ such that in $|\Re z|,|\Im z| \leq \delta$ no other zeros exist. For an arbitrary constant $c=c(N, k, \delta)>0$, we can choose $0<c_{1}<c_{2}<\delta$ such that for $|\Im z| \geq \delta$ :

$$
\left|\arg z^{2 n} \pm n \pi\right|<\frac{\pi}{2} \text { for } 1 \leq n \leq c|z|, c_{1}<\Re z<c_{2}
$$

Here the sign + is used if $\Im z>0$ and - if $\Im z<0$. The sum $2^{N / 2} F(z)$ is divided into two parts. For the indices $n \leq c|z|$ the real parts of the summands have the same sign, hence

$$
\begin{aligned}
& \left|\sum_{k \leq n \leq c|z|} \frac{(-1)^{n} a_{n}}{2^{2 n} n ! \Gamma(N / 2+n+1)} z^{2 n}\right| \geq \\
& \quad \geq\left|\Re \sum_{k \leq n \leq c|z|} \frac{(-1)^{n} a_{n} z^{2 n}}{2^{2 n} n ! \Gamma(N / 2+n+1)}\right| \\
& \quad \geq \frac{(2 k) !}{2^{2 k} k ! \Gamma(N / 2+k+1)} \Re z^{2 k}>\frac{(2 k) !}{2^{2 k} k ! \Gamma(N / 2+k+1)} \frac{1}{2} \delta^{2 k} .
\end{aligned}
$$




\section{Horváth}

On the other hand if $c$ is sufficiently large, then

$$
\begin{aligned}
& \left|\sum_{n \geq c|z|} \frac{(-1)^{n} a_{n} z^{2 n}}{2^{2 n} n ! \Gamma(N / 2+n+1)}\right| \leq \\
& \quad \leq \sum_{n \geq c|z|} \frac{2^{2 k} k^{2 n}}{2^{2 n}(n !)^{2}}|z|^{2 n}=2^{2 k} \sum_{n \geq c|z|}\left|\frac{k z}{2}\right|^{2 n}(n !)^{-2}< \\
& \quad<2^{2 k} \sum_{n \geq c|z|}\left|\frac{k z e}{2 n}\right|^{2 n}<\frac{(2 k) ! \delta^{2 k}}{4 \cdot 2^{2 k} k ! \Gamma(N / 2+k+1)}
\end{aligned}
$$

which shows that $|F(z)|$ is bounded below in the whole strip, so (11) holds indeed. Lemma 4 is proved.

LEMMA 5.- Let $f \in C_{0}^{\infty}(\Omega)$, then

$$
\sigma_{\lambda}(f, x) \longrightarrow f(x)
$$

locally uniformly in $\Omega$.

Proof. - We start with the square sum estimate

$$
\sum_{\left|\mu-\rho_{n}\right| \leq 1}\left|u_{n}(x)\right|^{2} e^{4\left|\nu_{n}\right| R} \leq c \mu^{N-1}, \quad x \in K \subset \Omega, \mu \geq 1
$$

which holds uniformly on the compact set $K$ and in $\mu$ under the weaker condition that $\left(u_{n}\right)$ is a Bessel system of eigenfunctions and

$$
0<R<\min \left\{\frac{1}{2} \operatorname{dist}(K, \partial \Omega), \frac{\pi}{3}\right\}
$$

is arbitrarily chosen ([6], [9]). Since $f \in H_{2}^{\alpha}\left(\mathbb{R}^{N}\right)$ for any $\alpha>0$, we see for $\alpha>N / 2$ that

$$
\begin{aligned}
& \left|\sum_{\rho_{n} \geq \lambda}\left\langle f, v_{n}\right\rangle u_{n}(x)\right| \leq \\
& \leq \sum_{k=0}^{\infty} \sum_{2^{k} \lambda \leq \rho_{n} \leq 2^{k+1} \lambda}\left|\left\langle f, v_{n}\right\rangle\right|\left|u_{n}(x)\right|
\end{aligned}
$$


Local uniform convergence of the Riesz means of Laplace and Dirac expansions

$$
\begin{aligned}
& \leq c \sum_{k=0}^{\infty}\left(\sum_{2^{k} \lambda \leq \rho_{n} \leq 2^{k+1} \lambda}\left|\left\langle f, v_{n}\right\rangle\right|^{2} \rho_{n}^{2 \alpha}\right)^{1 / 2}\left(\sum_{2^{k} \lambda \leq \rho_{n} \leq 2^{k+1} \lambda} \frac{\left|u_{n}(x)\right|^{2}}{\left(2^{k} \lambda\right)^{2 \alpha}}\right)^{1 / 2} \\
& \leq c(K)\|f\|_{H_{2}^{\alpha}} \sum_{k=0}^{\infty}\left(2^{k} \lambda \cdot\left(2^{k} \lambda\right)^{N-1} \cdot\left(2^{k} \lambda\right)^{-2 \alpha}\right)^{1 / 2} \\
& \leq c(K)\|f\|_{H_{2}^{\alpha}} \cdot \lambda^{N / 2-\alpha} \sum_{k=0}^{\infty} 2^{k(N / 2-\alpha)} \leq c(K)\|f\|_{H_{2}^{\alpha}} \cdot \lambda^{N / 2-\alpha}
\end{aligned}
$$

which proves Lemma 5.

Remark. - In fact we proved that the expansion of any $f \in H_{2}^{\alpha}\left(\mathbb{R}^{N}\right)$, supp $f \subset \Omega$ converges absolutely and locally uniformly. The same statement can be proved in case $f \in H_{p}^{\alpha}\left(\mathbb{R}^{N}\right)$, supp $f \subset \Omega, 1<p<\infty, \alpha>N / 2$, $\alpha p>N$. Indeed, the case $p=2$ was done above; if $p>2$ then $f \in H_{p}^{\alpha}$ implies $f \in H_{2}^{\alpha}$ by the boundedness of $\Omega$. In case $p<2$, we use the imbedding (5); it makes possible to increase the value $p$ until the bound $q<q_{0}$,

$$
\alpha-N\left(\frac{1}{p}-\frac{1}{q_{0}}\right)=\frac{N}{2}, \quad \frac{1}{q_{0}}=\frac{1}{2}-\frac{\alpha}{N}+\frac{1}{p}=\frac{1}{2}+\frac{N-\alpha p}{N p}<\frac{1}{2}
$$

hence we have $H_{p}^{\alpha} \subset H_{2}^{\beta}$ for some $\beta>N / 2$ which finishes the proof.

Proof of Theorem 1. - We can diminish the value $s$ in case $s \geq(N-1) / 2$ to ensure the additional condition $s<(N-1) / 2$. Consider the imbedding $\stackrel{\circ}{H}_{p}^{\alpha}(\Omega) \subset \stackrel{\circ}{H}_{q}^{\beta}(\Omega)$ given in Lemma 3 ; it is enough to prove Theorem 1 for $f \in \stackrel{\circ}{H}_{q}^{\beta}(\Omega)$, supp $f \subset \Omega$. The following auxiliary statement is true: given a function $\tau \in C_{0}^{\infty}\left(\mathbb{R}^{N}\right)$, the operator

$$
f \longmapsto \tau f
$$

is continuous in $H_{p}^{\alpha}\left(\mathbb{R}^{N}\right)$. Indeed, we have $\|\tau f\|_{L_{p}} \leq c\|f\|_{L_{p}}$ and for $\alpha=\ell+\chi, 0<\chi \leq 1, \ell \in \mathbb{N}, k=2$ and for any multiindex $\beta,|\beta|=\ell$, 


$$
\begin{aligned}
& \left\|\Delta_{h}^{2}\left((\tau f)^{(\beta)}\right)\right\|_{L_{p}} \leq \\
& \leq c \sum_{\gamma+\delta=\beta}\left\|\Delta_{h}^{2}\left(\tau^{(\gamma)} f^{(\delta)}\right)\right\|_{L_{p}} \\
& \leq c \sum_{\gamma+\delta=\beta}\left\{\left\|\tau^{(\gamma)} \Delta_{h}^{2} f^{(\delta)}\right\|_{L_{p}}+\right. \\
& \quad+\left\|\left(\tau^{(\gamma)}(x+2 h)-\tau^{(\gamma)}(x)\right) f^{(\delta)}(x+2 h)\right\|_{L_{p}} \\
& \left.\quad+\left\|\left(\tau^{(\gamma)}(x+h)-\tau^{(\gamma)}(x)\right) f^{(\delta)}(x+h)\right\|_{L_{p}}\right\} \\
& \leq c \sum_{\gamma+\delta=\beta}\left(\left\|\Delta_{h}^{2} f^{(\delta)}\right\|_{L_{p}}+h\left\|f^{(\delta)}\right\|_{L_{p}}\right) \\
& \leq c \sum_{\gamma+\delta=\beta}\left(h^{\chi}\|f\|_{H_{p}^{|\delta|+\chi}}+h\|f\|_{L_{p}^{|\delta|}}\right) \leq c h^{\chi}\|f\|_{H_{p}^{\alpha}}
\end{aligned}
$$

(here $L_{p}^{\alpha}$ denotes the Liouville class of indices $p, \alpha ;[21]$ ). So the mapping $f \mapsto \tau f$ is continuous indeed. Now let $f \in \stackrel{\circ}{H}_{q}^{\beta}(\Omega)$, then there exist $f_{n} \in C_{0}^{\infty}(\Omega)$ tending to $f$ in $H_{q}^{\beta}$-norm. By the above proved statement, we can suppose that there is a compact set $K \subset \Omega$ such that supp $f_{n} \subset K$ for all $n$. Then we apply Lemma 2 .

$$
\begin{aligned}
& \left|f(x)-\sigma_{\lambda}^{s}(f, x)\right| \leq \\
& \leq\left|f(x)-f_{n}(x)\right|+\left|\sigma_{\lambda}^{s}\left(f-f_{n}, x\right)\right|+\left|f_{n}(x)-\sigma_{\lambda}^{s}\left(f_{n}, x\right)\right|=: I_{1}+I_{2}+I_{3} .
\end{aligned}
$$

Taking an arbitrary $\varepsilon>0$, we see

$$
I_{2} \leq c\left\|f-f_{n}\right\|_{H_{q}^{\beta}}<\varepsilon, \quad n>n(\varepsilon)
$$

independently of $\lambda$. From $\beta q>N$ it follows that $H_{q}^{\beta} \subset C^{t}\left(\mathbb{R}^{N}\right)$, $0<t<\beta-N / q[24, \S 2.7 .1]$; in particular

$$
I_{1} \leq c\left\|f-f_{n}\right\|_{H_{q}^{\beta}}<\varepsilon, \quad n>n(\varepsilon) .
$$

Fix an index $n>n(\varepsilon)$ and let $\lambda$ tend to infinity; by Lemma 5 for large $\lambda$, we have

$$
I_{3}<\varepsilon
$$

and then $\left|f(x)-\sigma_{\lambda}^{s}(f, x)\right|<3 \varepsilon$ for $\lambda$ large enough. The proof is complete. 
Local uniform convergence of the Riesz means of Laplace and Dirac expansions

Proof of Theorem 2.- If $s \geq(N-1) / 2$, we diminish $s$ to obtain $s<(N-1) / 2, \alpha+s \geq(N-1) / 2$. Then we diminish $\alpha$ until $\alpha+s=(N-1) / 2$, then $\stackrel{\circ}{\mathrm{O}}_{2}^{\alpha}$ is growing. Let $x_{0} \in \Omega \backslash \operatorname{supp} f, \operatorname{dist}\left(x_{0}, \operatorname{supp} f\right)>\rho>0$. Since $f \in \stackrel{\circ}{H}_{2}^{\alpha}, f$ can be approximated in $H_{2}^{\alpha}$-norm by functions $f_{n} \in C_{0}^{\infty}(\Omega)$, $\operatorname{dist}\left(x_{0}, \operatorname{supp} f_{n}\right)>\rho$. We fix the value $\rho$ and let tend $\lambda$ to infinity; for $\lambda \geq 4 \pi / \rho$, Lemma 1 gives the boundedness of $\sigma_{\lambda}^{s}\left(f, x_{0}\right)$. We have

$$
\left|\sigma_{\lambda}^{s}\left(f, x_{0}\right)\right| \leq\left|\sigma_{\lambda}^{s}\left(f-f_{n}, x_{0}\right)\right|+\left|\sigma_{\lambda}^{s}\left(f_{n}, x_{0}\right)\right|=: I_{1}+I_{2} .
$$

For $\lambda>1 / \rho$, we have

$$
I_{1} \leq c\left\|f-f_{n}\right\|_{H_{2}^{\alpha}}<\varepsilon \text { if } n>n(\varepsilon) .
$$

We fix such an $n$; then for large $\lambda$, we have $I_{2}<\varepsilon$ which proves Theorem 2 .

\section{Technical background of the proof of Lemma 1}

We showed above the Theorems through Lemma 1. In this section we give some estimates and an expression for $\sigma_{\lambda}^{s}\left(f, x_{0}\right)$ which make possible to prove Lemma 1. The proof will be finished in the fourth section.

Define the operators

$$
\mathrm{D} F(r):=\left(\frac{1}{r} F(r)\right)^{\prime}, \quad \mathrm{D}^{k} F(r):=\mathrm{D}\left(\mathrm{D}^{k-1} F(r)\right)
$$

and the functions

$$
\begin{aligned}
\psi(r) & :=\int_{\theta} f\left(x_{0}+r \theta\right) \mathrm{d} \theta \\
F(r) & :=\frac{r^{N-1}}{\omega_{N}} \int_{\theta} f\left(x_{0}+r \theta\right) \mathrm{d} \theta
\end{aligned}
$$

where $\omega_{N}$ is the surface of the unit ball.

LEMMA 6. - Let $f \in \stackrel{\circ}{H}_{2}^{\alpha}(\Omega)$ and suppose that the conditions of Lemma 1 hold. Let $\alpha=\ell+\chi, 0<\chi \leq 1, \ell \in \mathbb{N}$. Then:

a) the function $\psi(r)$ can be modified on a set of measure zero such that the new function, denoted again by $\psi$ and its derivatives $\psi^{\prime}, \psi^{\prime \prime}, \ldots, \psi^{(\ell-1)}$ are locally absolutely continuous and

$$
\psi^{(k)}(r)=\int_{\theta} f^{(k)}\left(x_{0}+r \theta\right) \mathrm{d} \theta \quad \text { a.e., } 0 \leq k \leq \ell
$$


where

$$
f^{(k)}\left(x_{0}+r \theta\right):=\sum_{|\beta|=k} f^{(\beta)}\left(x_{0}+r \theta\right) \cdot \theta^{\beta}
$$

$\theta^{\beta}=\theta_{1}^{\beta_{1}} \ldots \theta_{N}^{\beta_{N}}$, where $\theta=\left(\theta_{1}, \ldots, \theta_{N}\right) \in S_{N-1} ;$

b) $\mathrm{D}^{k} F(r)=\frac{(2 \pi)^{N / 2}}{\omega_{N}} \sum\left\langle f, v_{n}\right\rangle u_{n}\left(x_{0}\right) r^{N-2 k-1} \frac{J_{N / 2-k-1}\left(\mu_{n} r\right)}{\left(\mu_{n} r\right)^{N / 2-k-1}}$, $k=0,1, \ldots, \ell-1$.

Proof. - Since $H_{2}^{\alpha} \subset L_{2}^{\ell}$, we prove the point a) for $f \in L_{2}^{\ell}\left(\mathbb{R}^{N}\right)$. Because $C_{0}^{\infty}\left(\mathbb{R}^{N}\right)$ is dense in $L_{2}^{\ell}\left(\mathbb{R}^{N}\right)$, there exist $f_{n} \in C_{0}^{\infty}$ with $f_{n} \rightarrow f$ in $L_{2}^{\ell}$. Since the multiplication by some $\tau \in C_{0}^{\infty}$ is obviously continuous, we can suppose that

$$
\operatorname{supp} f_{n} \subset K, \quad \operatorname{dist}\left(x_{0}, \operatorname{supp} f_{n}\right)>\frac{\rho}{2} \quad \text { for all } n .
$$

We know that

$$
\frac{\mathrm{d}}{\mathrm{d} r} \int_{\theta} f_{n}^{(k)}\left(x_{0}+r \theta\right) \mathrm{d} \theta=\int_{\theta} f_{n}^{(k+1)}\left(x_{0}+r \theta\right) \mathrm{d} \theta
$$

and then by (16) we obtain for $n \rightarrow \infty$ and $k<\ell$

$$
\begin{aligned}
& \int_{\theta} f_{n}^{(k)}\left(x_{0}+r \theta\right) \mathrm{d} \theta=\int_{0}^{r} \int_{\theta} f_{n}^{(k+1)}\left(x_{0}+t \theta\right) \mathrm{d} \theta \mathrm{d} t= \\
& =\int_{|u| \leq r}|u|^{1-N} f_{n}^{(k+1)}\left(x_{0}+u\right) \mathrm{d} u \longrightarrow \\
& \quad \longrightarrow \int_{|u| \leq r}|u|^{1-N} f^{(k+1)}\left(x_{0}+u\right) \mathrm{d} u=\int_{0}^{r} \int_{\theta} f^{(k+1)}\left(x_{0}+t \theta\right) \mathrm{d} \theta \mathrm{d} t .
\end{aligned}
$$

On the other hand

$$
\begin{aligned}
& \int_{0}^{R}\left|\int_{\theta} f_{n}^{(k)}\left(x_{0}+r \theta\right) \mathrm{d} \theta-\int_{\theta} f^{(k)}\left(x_{0}+r \theta\right) \mathrm{d} \theta\right| \mathrm{d} r \leq \\
& \leq \int_{|u| \leq R}\left|f_{n}^{(k)}\left(x_{0}+u\right)-f^{(k)}\left(x_{0}+u\right)\right| \cdot|u|^{1-N} \mathrm{~d} u \leq c(\rho)\left\|f_{n}^{(k)}-f^{(k)}\right\|_{L_{2}} \\
& \leq c(\rho) \sum_{|\beta|=k}\left\|f_{n}^{(\beta)}-f^{(\beta)}\right\|_{L_{2}} \stackrel{n \rightarrow \infty}{\longrightarrow} 0
\end{aligned}
$$


Local uniform convergence of the Riesz means of Laplace and Dirac expansions hence taking an appropriate subsequence

$$
\int_{\theta} f_{n}^{(k)}\left(x_{0}+r \theta\right) \mathrm{d} \theta \longrightarrow \int_{\theta} f^{(k)}\left(x_{0}+r \theta\right) \mathrm{d} \theta \quad \text { a.e. }
$$

and then

$$
\int_{\theta} f^{(k)}\left(x_{0}+r \theta\right) \mathrm{d} \theta=\int_{0}^{r} \int_{\theta} f^{(k+1)}\left(x_{0}+t \theta\right) \mathrm{d} \theta \mathrm{d} t \quad \text { a.e. }
$$

So if we modify $\psi$ on a null-set by $\psi=\iint \cdots \int \psi^{(\ell)}$, the statement a) is proved. To see $\mathrm{b}$ ), denote $A_{k}(r)$ the right hand sum of $\left.\mathrm{b}\right)$. First we show that the sum $A_{k}$ converges absolutely and uniformly in $r \in[0, R]$. Indeed,

$$
\begin{aligned}
& \left|\sum_{\left|\mu_{n}\right| \leq 1}\right| \leq \\
& \quad \leq c \sum_{\left|\mu_{n}\right| \leq 1}\left|\left\langle f, v_{n}\right\rangle\right|\left|u_{n}\left(x_{0}\right)\right| r^{N-2 k-1} \\
& \quad \leq c\left(\sum_{\left|\mu_{n}\right| \leq 1}\left|\left\langle f, v_{n}\right\rangle\right|^{2}\right)^{1 / 2}\left(\sum_{\rho_{n} \leq 1}\left|u_{n\left(x_{0}\right)}\right|^{2}\right)^{1 / 2} \leq c\|f\|_{L_{2}} . \\
& \sum_{\rho_{n} \leq 1 \leq\left|\mu_{n}\right|}\left|\leq \sum_{\rho_{n} \leq 1 \leq\left|\mu_{n}\right|}\right|\left\langle f, v_{n}\right\rangle|| u_{n}\left(x_{0}\right) \mid \frac{r^{N / 2-k}}{\left|\mu_{n}\right|^{N / 2-k-1}} \mathrm{e}^{\left|\nu_{n}\right| R}\left(\left|\mu_{n} r\right|\right)^{-1 / 2} \\
& \leq c \sum_{\rho_{n} \leq 1}\left|\left\langle f, v_{n}\right\rangle\right|\left|u_{n}\left(x_{0}\right)\right| \mathrm{e}^{\left|\nu_{n}\right| R} \\
& \leq c\left(\sum_{\left|\rho_{n}\right| \leq 1}\left|\left\langle f, v_{n}\right\rangle\right|^{2}\right)^{1 / 2}\left(\sum_{\rho_{n} \leq 1}\left|u_{n}\left(x_{0}\right)\right|^{2} \mathrm{e}^{2\left|\nu_{n}\right| R}\right)^{1 / 2} \leq c\|f\|_{L_{2}}, \\
& \quad-667-
\end{aligned}
$$


M. Horváth

$$
\begin{aligned}
& \left|\sum_{2^{m} \leq \rho_{n} \leq 2^{m+1}}\right| \leq \\
& \leq c \sum\left|\left\langle f, v_{n}\right\rangle\right|\left|u_{n}\left(x_{0}\right)\right| \mathrm{e}^{\left|\nu_{n}\right| R} \rho_{n}^{k-(N-1) / 2} \\
& \leq c\left(\sum_{2^{m} \leq \rho_{n} \leq 2^{m+1}}\left|\left\langle f, v_{n}\right\rangle\right|^{2} \rho_{n}^{2 \alpha}\right)^{1 / 2}\left(\sum_{2^{m} \leq \rho_{n} \leq 2^{m+1}} \frac{\left|u_{n}\left(x_{0}\right)\right|^{2} \mathrm{e}^{2\left|\nu_{n}\right| R}}{\rho_{n}^{N-1-2 k+2 \alpha}}\right)^{1 / 2} \\
& \leq c\|f\|_{H_{2}^{\alpha}}\left(2^{m N} 2^{-(N-1-2 k+2 \alpha) m}\right)^{1 / 2}=c\|f\|_{H_{2}^{\alpha}} 2^{m(k+1 / 2-\alpha)}
\end{aligned}
$$

and then

$$
\left|A_{k}\right|=c\left(\|f\|_{L_{2}}+\|f\|_{H_{2}^{\alpha}} \sum_{m=0}^{\infty} 2^{m(k+1 / 2-\alpha)}\right) \leq c\|f\|_{H_{2}^{\alpha}}
$$

By the rule

$$
\int r^{\nu+1} J_{\nu}(\mu r) \mathrm{d} r=\frac{1}{\mu} r^{\nu+1} J_{\nu+1}(\mu r)
$$

this implies that

$$
\frac{\mathrm{d}}{\mathrm{d} r}\left(\frac{1}{r} A_{k}(r)\right)=A_{k+1}(r), \quad k=0,1, \ldots, \ell-2 .
$$

From (8), we get that

$$
\begin{aligned}
\int_{0}^{r} F(t) \mathrm{d} t & =\frac{1}{\omega_{N}} \int_{|z| \leq r} f\left(x_{0}+z\right) \mathrm{d} z \\
& =\frac{(2 \pi)^{N / 2}}{\omega_{N}} \sum\left\langle f, v_{n}\right\rangle u_{n}\left(x_{0}\right)\left(\frac{r}{\mu_{n}}\right)^{N / 2} J_{N / 2}\left(\mu_{n} r\right) .
\end{aligned}
$$

The differentiated series is that of $A_{0}(r)$ and converges uniformly; hence $F(r)=A_{0}(r)$ and then $\mathrm{D}^{k} F(r)=A_{k}(r)$. Lemma 6 is proved.

\section{LEMMa 7}

a) Let $\varphi_{k}(r):=r^{\chi+k-1} \psi^{(k)}(r), 0 \leq k \leq \ell$, and suppose $\lambda \rho \geq 4 \pi$. Then, for every $\varepsilon>0$,

$$
\int_{\rho}^{R}\left|2 \varphi_{k}(r)-\varphi_{k}\left(r+\frac{\pi}{\lambda}\right)-\varphi_{k}\left(r-\frac{\pi}{\lambda}\right)\right| \mathrm{d} r \leq c \lambda^{-\chi} \rho^{\alpha-N / 2-\varepsilon}\|f\|_{H_{2}^{\alpha}} .
$$


Local uniform convergence of the Riesz means of Laplace and Dirac expansions

b) For every $\varepsilon>0$,

$$
\lambda^{N / 2-s-\ell}\left|\int_{0}^{R} \mathrm{D}^{\ell} F(r) \frac{J_{N / 2+s-\ell}(\lambda r)}{r^{N / 2+s-\ell}} \mathrm{d} r\right| \leq c \rho^{\alpha-N / 2-\varepsilon}\|f\|_{H_{2}^{\alpha}}
$$

whenever $R$ satisfies

$$
\cos \left(\lambda R-\frac{\pi}{2}\left(\frac{N+1}{2}+1+s-\ell\right)\right)=0 .
$$

Proof. - Let $2<p<\infty$ be defined by

$$
\alpha-k-\varepsilon=N\left(\frac{1}{2}-\frac{1}{p}\right) ;
$$

then the imbeddings

$$
H_{2}^{\alpha-k}\left(\mathbb{R}^{N}\right) \subset H_{p}^{\varepsilon}\left(\mathbb{R}^{N}\right) \subset L_{p}\left(\mathbb{R}^{N}\right)
$$

hold. If we change $(r \pm \pi / \lambda)^{\chi+k-1}$ by $r^{\chi+k-1}$, the error is

$$
\left(r \pm \frac{\pi}{\lambda}\right)^{\chi+k-1}-r^{\chi+k-1}=\mathrm{O}\left(\frac{1}{\lambda}\left(r \pm \frac{\pi}{\lambda}\right)^{\chi+k-2}\right)
$$

hence by the Hölder inequality

$$
\begin{aligned}
& \int_{\rho}^{R}\left|\left(\left(r \pm \frac{\pi}{\lambda}\right)^{\chi+k-1}-r^{\chi+k-1}\right) \psi^{(k)}\left(r \pm \frac{\pi}{\lambda}\right)\right| \mathrm{d} r \leq \\
& \leq \frac{c}{\lambda} \int_{\rho}^{R}\left(r \pm \frac{\pi}{\lambda}\right)^{\chi+k-2}\left|\psi^{(k)}\left(r \pm \frac{\pi}{\lambda}\right)\right| \mathrm{d} r \\
& \leq \frac{c}{\lambda}\left(\int_{\rho}^{R+\pi / \lambda} r^{N-1}\left|\psi^{(k)}(r)\right|^{p} \mathrm{~d} t\right)^{1 / p}\left(\int_{\rho / 2}^{R} r^{(\chi+k-2-(N-1) / p) q} \mathrm{~d} r\right)^{1 / q} \\
& \leq \frac{c}{\lambda}\left(\int_{\rho \leq|u| \leq R+\pi / \lambda}\left|f^{(k)}\left(x_{0}+u\right)\right|^{p} \mathrm{~d} u\right)^{1 / p} \rho^{\chi+k-2-(N-1) / p+1 / q} \\
& \leq \frac{c}{\lambda}\left\|f^{(k)}\right\|_{L_{p}} \rho^{\alpha-N / 2-1+\chi-\varepsilon} \leq \frac{c}{\lambda} \rho^{\alpha-N / 2-1+\chi-\varepsilon} \sum_{|\beta|=k}\left\|f^{(\beta)}\right\|_{L_{p}} \\
& \leq c\|f\|_{H_{2}^{\alpha} \lambda^{-\chi} \rho^{\alpha-N / 2-\varepsilon}(\lambda \rho)^{\chi-1} \leq c\|f\|_{H_{2}^{\alpha} \lambda^{-\chi} \rho^{\alpha-N / 2-\varepsilon} .}}^{-669-}
\end{aligned}
$$




\section{Horváth}

Hence to prove a) it is enough to show that

$$
\begin{aligned}
& \int_{\rho}^{R} r^{\chi+k-1}\left|2 \psi^{(k)}(r)-\psi^{(k)}\left(r+\frac{\pi}{\lambda}\right)-\psi^{(k)}\left(r-\frac{\pi}{\lambda}\right)\right| \mathrm{d} r \leq \\
& \quad \leq c \lambda^{-\chi} \rho^{\alpha-N / 2-\varepsilon}\|f\|_{H_{2}^{\alpha}} .
\end{aligned}
$$

To this we use the following characterization of $H_{p}^{\alpha}, 1<p<\infty, \alpha>0$ (see [21] or $[24, \S 2.3 .1]$ with the notation $\left.B_{p, \infty}^{\alpha}\left(\mathbb{R}^{N}\right)\right)$ : the function $f$ belongs to $H_{p}^{\alpha}\left(\mathbb{R}^{N}\right)$ if and only if it has a representation

$$
f(x)=\sum_{m=1}^{\infty} Q_{m}(x), \quad\left\|Q_{m}\right\|_{L_{p}} \leq c 2^{-m \alpha}\|f\|_{H_{p}^{\alpha}}
$$

where the $Q_{m}$ are entire function of spherical exponential type $\leq 2^{m}$; in other words, the Fourier transform has bounded support:

$$
\operatorname{supp} F Q_{m} \subset\left(|\xi| \leq 2^{m}\right)
$$

We shall also use the Bernstein type inequality

$$
\left\|Q_{m}^{(\beta)}\right\|_{L_{p}} \leq 2^{m|\beta|}\left\|Q_{m}\right\|_{L_{p}}
$$

For our purpose it is more convenient to take a new $p$ defined by

$$
\alpha-k-\chi=N\left(\frac{1}{2}-\frac{1}{p}\right)
$$

it means the imbedding

$$
H_{2}^{\alpha-k} \subset H_{p}^{\chi}
$$

If $|\beta|=k, f^{(\beta)} \in H_{2}^{\alpha-k} \subset H_{p}^{\chi}$ has a representation

$$
f^{(\beta)}(x)=\sum_{m} Q_{m, \beta}(x), \quad\left\|Q_{m, \beta}\right\|_{L_{p}} \leq c 2^{-m \chi}\left\|f^{(\beta)}\right\|_{H_{p}^{\chi}} \leq c 2^{-m \chi}\|f\|_{H_{2}^{\alpha}}
$$


Local uniform convergence of the Riesz means of Laplace and Dirac expansions

hence

$$
\begin{aligned}
& \left(\int_{\rho}^{R} r^{N-1} \mid \int_{\theta}\left(2 f^{(k)}\left(x_{0}+r \theta\right)+\right.\right. \\
& \left.\left.\quad-f^{(k)}\left(x_{0}+\left(r+\frac{\pi}{\lambda}\right) \theta\right)-f^{(k)}\left(x_{0}+\left(r-\frac{\pi}{\lambda}\right) \theta\right)\right)\left.\mathrm{d} \theta\right|^{p} \mathrm{~d} r\right)^{1 / p} \leq \\
& \leq c \sum_{|\beta|=k}\left(\int_{\rho}^{R} r^{N-1} \int_{\theta} \mid 2 f^{(\beta)}\left(x_{0}+r \theta\right)-f^{(\beta)}\left(x_{0}+\left(r+\frac{\pi}{\lambda}\right) \theta\right)+\right. \\
& \left.\quad-\left.f^{(\beta)}\left(x_{0}+\left(r-\frac{\pi}{\lambda}\right) \theta\right)\right|^{p} \mathrm{~d} \theta \mathrm{d} r\right)^{1 / p} \\
& \leq c \sum_{|\beta|=k} \sum_{m=1}^{\infty}\left(\int_{\rho \leq|u| \leq R} \mid 2 Q_{m, \beta}\left(x_{0}+u\right)-Q_{m, \beta}\left(x_{0}+u\left(1+\frac{\pi}{\lambda r}\right)\right)+\right. \\
& \left.\quad-\left.Q_{m, \beta}\left(x_{0}+u\left(1-\frac{\pi}{\lambda r}\right)\right)\right|^{p} \mathrm{~d} u\right)^{1 / p} .
\end{aligned}
$$

Let $m^{*}$ be an integer to be precised later. The sum $\sum_{m>m^{*}}$ can be estimated by

$$
c \sum_{m>m^{*}}\left\|Q_{m, \beta}\right\|_{L_{p}} \leq c\left\|f^{(\beta)}\right\|_{H_{p}^{\chi}} \sum_{m>m^{*}} 2^{-m \chi} \leq c\left\|f^{(\beta)}\right\|_{H_{2}^{\alpha}} 2^{-m^{*} \chi}
$$

and the sum $\sum_{m \leq m^{*}}$ by

$$
\begin{aligned}
& \sum_{m \leq m^{*}}\left(\int_{\rho}^{R} r^{N-1} \times\right. \\
& \left.\quad \times \int_{\theta}\left|\int_{0}^{\pi / \lambda} \int_{-\pi / \lambda}^{0} \frac{\partial^{2}}{\partial r^{2}} Q_{m, \beta}\left(x_{0}+\left(r+t_{1}+t_{2}\right) \theta\right) \mathrm{d} t_{2} \mathrm{~d} t_{1}\right|^{p} \mathrm{~d} \theta \mathrm{d} r\right)^{1 / p} \leq \\
& \leq c \sum_{m \leq m^{*}}\left(\int_{\rho}^{R} r^{N-1} \int_{\theta} \lambda^{-2 p / q} \int_{0}^{\pi / \lambda} \times\right. \\
& \left.\quad \times \int_{-\pi / \lambda}^{0}\left|\frac{\partial^{2}}{\partial r^{2}} Q_{m, \beta}\left(x_{0}+\left(r+t_{1}+t_{2}\right) \theta\right)\right|^{p} \mathrm{~d} t_{2} \mathrm{~d} t_{1} \mathrm{~d} \theta \mathrm{d} r\right)^{1 / p}
\end{aligned}
$$




$$
\begin{aligned}
& \leq c \sum_{m \leq m^{*}}\left(\int_{0}^{\pi / \lambda} \int_{-\pi / \lambda}^{0} \lambda^{-2 p / q} \int_{\rho}^{R} r^{N-1} \times\right. \\
&\left.\times \int_{\theta}\left|\frac{\partial^{2}}{\partial r^{2}} Q_{m, \beta}\left(x_{0}+\left(r+t_{1}+t_{2}\right) \theta\right)\right|^{p} \mathrm{~d} \theta \mathrm{d} r \mathrm{~d} t_{2} \mathrm{~d} t_{1}\right)^{1 / p} \\
& \leq c \sum_{m \leq m^{*}}\left(\int_{0}^{\pi / \lambda} \int_{-\pi / \lambda}^{0} \lambda^{-2 p / q}\left\|\frac{\partial}{\partial r^{2}} Q_{m, \beta}\right\|_{L_{p}}^{p} \mathrm{~d} t_{2} \mathrm{~d} t_{1}\right)^{1 / p} \\
& \leq c \lambda^{-2} \sum_{m \leq m^{*}}\left(2^{2 m p}\left\|Q_{m, \beta}\right\|_{L_{p}}^{p}\right)^{1 / p} \\
& \leq c \lambda^{-2} \sum_{m \leq m^{*}}\left(2^{p m(2-\chi)}\|f\|_{H_{2}^{\alpha}}^{p}\right)^{1 / p} \leq c \lambda^{-2} 2^{m^{*}(2-\chi)}\|f\|_{H_{2}^{\alpha}} .
\end{aligned}
$$

Choose $m^{*}$ such that $\lambda \leq 2^{m^{*}}<2 \lambda$, then the above counting gives that

$$
\begin{aligned}
& \left(\int_{\rho}^{R} r^{N-1}\left|2 \psi^{(k)}(r)-\psi^{(k)}\left(r+\frac{\pi}{\lambda}\right)-\psi^{(k)}\left(r-\frac{\pi}{\lambda}\right)\right|^{p} \mathrm{~d} r\right)^{1 / p} \leq \\
& \quad \leq c \lambda^{-\chi}\|f\|_{H_{2}^{\alpha}} .
\end{aligned}
$$

But then by the Hölder inequality, we obtain

$$
\begin{aligned}
& \int_{\rho}^{R} r^{\chi+k-1}\left|2 \psi^{(k)}(r)-\psi^{(k)}\left(r+\frac{\pi}{\lambda}\right)-\psi^{(k)}\left(r-\frac{\pi}{\lambda}\right)\right| \mathrm{d} r \leq \\
& \leq\left(\int_{\rho}^{R} r^{N-1}\left|2 \psi^{(k)}(r)-\psi^{(k)}\left(r+\frac{\pi}{\lambda}\right)-\psi^{(k)}\left(r-\frac{\pi}{\lambda}\right)\right|^{p} \mathrm{~d} r\right)^{1 / p} \times \\
& \quad \times\left(\int_{\rho}^{R} r^{(\chi+k-1-(N-1) / p) q} \mathrm{~d} r\right)^{1 / q} \\
& \leq c \lambda^{-\chi}\|f\|_{H_{2}^{\alpha}} \rho^{\alpha-N / 2}
\end{aligned}
$$

so (17) holds also with $\varepsilon=0$. Thus a) is proved and we turn to the estimate b). We see by induction on $\ell$ that there exist constants $c_{\ell, 0}, \ldots, c_{\ell, \ell}$ such that

$$
\begin{gathered}
\mathrm{D}^{\ell} F(r)=c_{\ell, 0} r^{N-2 \ell-1} \psi(r)+c_{\ell, 1} r^{N-2 \ell} \psi^{\prime}(r)+\cdots \\
\cdots+c_{\ell, \ell} r^{N-\ell-1} \psi^{(\ell)}(r) . \\
-672-
\end{gathered}
$$


Local uniform convergence of the Riesz means of Laplace and Dirac expansions

Consequently it is enough to show that

$$
\begin{gathered}
\lambda^{N / 2-s-\ell}\left|\int_{\rho}^{R} r^{N-2 \ell-1+k} \psi^{(k)}(r) \frac{J_{N / 2+s-\ell}(\lambda r)}{r^{N / 2+s-\ell}} \mathrm{d} r\right| \leq \\
\quad \leq c \rho^{\alpha-N / 2-\varepsilon}\|f\|_{H_{2}^{\alpha}}, \quad k=0,1, \ldots, \ell .
\end{gathered}
$$

Since $\lambda r \geq \lambda \rho \geq 4 \pi$, the argument of the Bessel function is not small. We recall the asymptotical formula

$$
J_{\nu}(x)=\left(\frac{2}{\pi x}\right)^{1 / 2} \cos \left(x-\frac{\pi}{2}\left(\nu+\frac{1}{2}\right)\right)+\mathrm{O}\left(x^{-3 / 2}\right) .
$$

We choose again $p$ by

$$
\alpha-k-\varepsilon=N\left(\frac{1}{2}-\frac{1}{p}\right)
$$

Putting the remainder term in (20), we get

$$
\begin{aligned}
& \lambda^{(N-3) / 2-s-\ell} \int_{\rho}^{R} r^{N-2 \ell-1+k-(N-3) / 2-s+\ell}\left|\psi^{(k)}(r)\right| \mathrm{d} r= \\
& =\lambda^{\chi-1} \int_{\rho}^{R} r^{\chi-2+k}\left|\psi^{(k)}(r)\right| \mathrm{d} r \leq \\
& \leq \lambda^{\chi-1}\left(\int_{\rho}^{R} r^{N-1}\left|\psi^{(k)}(r)\right|^{p} \mathrm{~d} r\right)^{1 / p}\left(\int_{\rho}^{R} r^{(\chi-2+k-(N-1) / p) q} \mathrm{~d} r\right)^{1 / q} \\
& \leq c \lambda^{\chi-1} \rho^{\chi-1+\alpha-\varepsilon-N / 2}\left\|f^{(k)}\right\|_{L_{p}} \\
& \leq c(\lambda \rho)^{\chi-1} \rho^{\alpha-N / 2-\varepsilon} \sum_{|\beta|=k}\left\|f^{(\beta)}\right\|_{L_{p}} \leq c \rho^{\alpha-N / 2-\varepsilon}\|f\|_{H_{2}^{\alpha}} .
\end{aligned}
$$

In the main term, we have to prove that

$$
\begin{gathered}
\lambda^{\chi}\left|\int_{\rho}^{R}{ }^{\chi+k-1} \psi^{(k)}(r) \cos (\lambda r+\gamma) \mathrm{d} r\right| \leq c \rho^{\alpha-N / 2-\varepsilon}\|f\|_{H_{2}^{\alpha}} \\
\gamma:=-\frac{\pi}{2}\left(\frac{N+1}{2}+s-\ell\right) . \\
-673-
\end{gathered}
$$


M. Horváth

Since

$$
\begin{aligned}
& \int_{R_{1}-2 \pi / \lambda}^{R_{1}} r^{\chi+k-1} \psi^{(k)}(r) \cos (\lambda r+\gamma) \mathrm{d} r= \\
& \quad=\int_{R_{1}-\pi / \lambda}^{R_{1}}\left(\varphi_{k}(r)-\varphi_{k}\left(r-\frac{\pi}{\lambda}\right)\right) \cos (\lambda r+\gamma) \mathrm{d} r \\
& \quad=\int_{R_{1}-2 \pi / \lambda}^{R_{1}-\pi / \lambda}\left(\varphi_{k}(r)-\varphi_{k}\left(r+\frac{\pi}{\lambda}\right)\right) \cos (\lambda r+\gamma) \mathrm{d} r
\end{aligned}
$$

hence we can write by $\rho \geq 4 \pi / \lambda$ that

$$
\begin{aligned}
& 2 \int_{\rho}^{R} r^{\chi+k-1} \psi^{(k)}(r) \cos (\lambda r+\gamma) \mathrm{d} r= \\
& =\int_{R-\pi / \lambda}^{R}+\left(\int_{R-2 \pi / \lambda}^{R}+\int_{R-3 \pi / \lambda}^{R-\pi / \lambda}\right)+\left(\int_{R-4 \pi / \lambda}^{R-2 \pi / \lambda}+\int_{R-5 \pi / \lambda}^{R-3 \pi / \lambda}\right)+\cdots \\
& \quad \cdots+\left(\int_{R-2(M+1) \pi / \lambda}^{R-2 M \pi / \lambda}+\int_{R-(2 M+3) \pi / \lambda}^{R-(2 M+1) \pi / \lambda}\right) \\
& =\int_{R-\pi / \lambda}^{R} r^{\chi+k-1} \psi^{(k)}(r) \cos (\lambda r+\gamma) \mathrm{d} r+ \\
& \quad+\left(\int_{R-2 \pi / \lambda}^{R-\pi / \lambda}+\int_{R-4 \pi / \lambda}^{R-3 \pi / \lambda}+\cdots+\int_{R-(2 M+2) \pi / \lambda}^{R-(2 M+1) \pi / \lambda}\right) \times \\
& \quad \times\left(2 \varphi_{k}(r)-\varphi_{k}\left(r+\frac{\pi}{\lambda}\right)-\varphi_{k}\left(r-\frac{\pi}{\lambda}\right)\right) \cos (\lambda r+\gamma) \mathrm{d} r:= \\
& :=I_{1}+I_{2} .
\end{aligned}
$$

Here we used the notation $M$ for the integer satisfying

$$
R-\frac{(2 M+2) \pi}{\lambda} \leq \rho<R-\frac{2 M \pi}{\lambda} .
$$

By the point a), we have

$$
\begin{gathered}
\left|I_{2}\right| \leq \int_{\rho}^{R}\left|2 \varphi_{k}(r)-\varphi_{k}\left(r+\frac{\pi}{\lambda}\right)-\varphi_{k}\left(r-\frac{\pi}{\lambda}\right)\right| \mathrm{d} r \\
\leq c \lambda^{-\chi} \rho^{\alpha-N / 2-\varepsilon}\|f\|_{H_{2}^{\alpha}} . \\
-674-
\end{gathered}
$$


Local uniform convergence of the Riesz means of Laplace and Dirac expansions

To estimate $I_{1}$ we have to suppose that $\cos (\lambda r+\gamma)$ vanishes at the centre of the integrational interval, i.e.,

$$
0=\cos \left(\lambda R-\frac{\pi}{2}+\gamma\right) \text {. }
$$

In this case

$$
\begin{aligned}
\left|I_{1}\right|= & \mid \int_{R-\pi /(2 \lambda)}^{R}\left(r^{\chi+k-1} \psi^{(k)}(r)+\right. \\
& \left.-\left(2 R-\frac{\pi}{\lambda}-r\right)^{\chi+k-1} \psi^{(k)}\left(2 R-\frac{\pi}{\lambda}-r\right)\right) \cos (\lambda r+\gamma) \mathrm{d} r \mid \leq \\
\leq & \int_{R-\pi /(2 \lambda)}^{R}\left|\varphi_{k}(r)-\varphi_{k}\left(2 R-\frac{\pi}{\lambda}-r\right)\right| \mathrm{d} r \\
\leq & \frac{c}{\lambda} \int_{R-\pi /(2 \lambda)}^{R}\left|\psi^{(k)}\left(2 R-\frac{\pi}{\lambda}-r\right)\right| \mathrm{d} r+ \\
& +c \int_{R-\pi /(2 \lambda)}^{R}\left|\psi^{(k)}(r)-\psi^{(k)}\left(2 R-\frac{\pi}{\lambda}-r\right)\right| \mathrm{d} r \\
= & I_{11}+I_{12} .
\end{aligned}
$$

Here we have

$$
I_{11} \leq c \lambda^{-1} \lambda^{-1 / 2}\left\|f^{(k)}\right\|_{L_{2}} \leq c \lambda^{-3 / 2}\|f\|_{H_{2}^{\alpha}} \leq c \lambda^{-\chi}\|f\|_{H_{2}^{\alpha}}
$$

and

$$
\begin{aligned}
& I_{12} \leq c \lambda^{-1 / 2}\left(\int_{R-\pi /(2 \lambda)}^{R} r^{N-1}\left|\psi^{(k)}(r)-\psi^{(k)}\left(2 R-\frac{\pi}{\lambda}-r\right)\right|^{2} \mathrm{~d} r\right)^{1 / 2} \\
& \leq c \lambda^{-1 / 2} \sum_{|\beta|=k}\left(\int_{R-\pi /(2 \lambda)}^{R} r^{N-1} \int_{\theta} \mid f^{\beta}\left(x_{0}+r \theta\right)+\right. \\
&\left.-\left.f^{(\beta)}\left(x_{0}+\left(2 R-\frac{\pi}{\lambda}-r\right) \theta\right)\right|^{2} \mathrm{~d} \theta \mathrm{d} r\right)^{1 / 2} \\
& \leq c \lambda^{-1 / 2} \sum_{|\beta|=k} \sum_{m=1}^{\infty}\left(\int_{R-\pi /(2 \lambda)}^{R} r^{N-1} \int_{\theta} \mid Q_{m, \beta}\left(x_{0}+r \theta\right)+\right. \\
&\left.-\left.Q_{m, \beta}\left(x_{0}+\left(2 R-\frac{\pi}{\lambda}-r\right) \theta\right)\right|^{2} \mathrm{~d} \theta \mathrm{d} r\right)^{1 / 2} \\
&-675-
\end{aligned}
$$


where $f^{(\beta)} \in H_{2}^{\alpha-k}, f^{(\beta)}=\sum Q_{m, \beta}$. Now for $m>m^{*}$, we have

$$
\sum_{m>m^{*}} \leq c \sum_{m>m^{*}}\left\|Q_{m, \beta}\right\|_{L_{2}} \leq c\|f\|_{H_{2}^{\alpha}} 2^{-m^{*}(\alpha-k)}
$$

and the other sum is

$$
\begin{aligned}
\sum_{m \leq m^{*}} \leq & c \sum_{m \leq m^{*}}\left(\int_{R-\pi /(2 \lambda)}^{R} r^{N-1} \times\right. \\
& \left.\times \int_{\theta}\left(\int_{0}^{\pi / \lambda}\left|\frac{\partial}{\partial r} Q_{m, \beta}\left(x_{0}+(r+t) \theta\right)\right| \mathrm{d} t\right)^{2} \mathrm{~d} \theta \mathrm{d} r\right)^{1 / 2} \\
\leq & c \sum_{m \leq m^{*}}\left(\int_{R-\pi /(2 \lambda)}^{R} r^{N-1} \times\right. \\
& \left.\times \int_{\theta} \lambda^{-1} \int_{0}^{\pi /(2 \lambda)}\left|\frac{\partial}{\partial r} Q_{m, \beta}\left(x_{0}+(r+t) \theta\right)\right|^{2} \mathrm{~d} t \mathrm{~d} \theta \mathrm{d} r\right)^{1 / 2} \\
\leq & c \sum_{m \leq m^{*}}\left(\lambda^{-1} \int_{0}^{\pi /(2 \lambda)}\left\|\frac{\partial}{\partial r} Q_{m, \beta}\right\|_{L_{2}}^{2} \mathrm{~d} t\right)^{1 / 2} \\
\leq & c \lambda^{-1} \sum_{m \leq m^{*}}\left(2^{2 m}\left\|Q_{m, \beta}\right\|_{L_{2}}^{2}\right)^{1 / 2} \leq c \lambda^{-1}\|f\|_{H_{2}^{\alpha}} \sum_{m \leq m^{*}} 2^{m(1+k-\alpha)} .
\end{aligned}
$$

Now in case $k<\ell$ choose $m^{*}=\infty$ and if $k=\ell$, let $m^{*}$ be the integer used in the proof of a). In both cases

$$
I_{12} \leq c \lambda^{-1 / 2} \ln \lambda \cdot \lambda^{-\chi}\|f\|_{H_{2}^{\alpha}} \leq c \lambda^{-\chi}\|f\|_{H_{2}^{\alpha}}
$$

which finishes the proof of Lemma 7 .

Denote for $\lambda>1$

$$
\sigma_{\lambda, 1}^{s}\left(f, x_{0}\right):=\sum_{\rho_{n}<1}\left\langle f, v_{n}\right\rangle u_{n}\left(x_{0}\right)\left(1-\rho_{n}^{2} \lambda^{-2}\right)^{s}
$$


Local uniform convergence of the Riesz means of Laplace and Dirac expansions

LeMma 8. - Let $\lambda>1, R>0,0 \leq s, f \in H_{2}^{\alpha}$, supp $f \subset \Omega$ and $f(x)=0$ for a.e. $\left|x-x_{0}\right|<\rho$. Then in case $s>0$

$$
\begin{aligned}
& \sigma_{\lambda}^{s}\left(f, x_{0}\right)=\sigma_{\lambda, 1}^{s}\left(f, x_{0}\right)+ \\
& +\frac{\Gamma(s+1) 2^{s}}{\lambda^{s+\ell-N / 2}} \sum_{\rho_{n} \geq 1}\left\langle f, v_{n}\right\rangle u_{n}\left(x_{0}\right) \int_{R}^{\infty} r^{-s} J_{N / 2+s-\ell}(\lambda r) \frac{J_{N / 2-\ell-1}\left(\rho_{n} r\right)}{\rho_{n}^{N / 2-\ell-1}} \mathrm{~d} r+ \\
& -\frac{\Gamma(s+1) 2^{s}}{\lambda^{s+\ell-N / 2}} \sum_{\rho_{n}<1}\left\langle f, v_{n}\right\rangle u_{n}\left(x_{0}\right) \int_{0}^{R} r^{-s} J_{N / 2+s-\ell}(\lambda r) \frac{J_{N / 2-\ell-1}\left(\mu_{n} r\right)}{\mu_{n}^{N / 2-\ell-1}} \mathrm{~d} r+ \\
& +\Gamma(s+1) 2^{s}(2 \pi)^{-N / 2} \omega_{N} \lambda^{N / 2-s-\ell} \int_{0}^{R} \mathrm{D}^{\ell} F(r) \frac{J_{N / 2+s-\ell}(\lambda r)}{r^{N / 2+s-\ell}} \mathrm{d} r+ \\
& -\Gamma(s+1) 2^{s} \lambda^{N / 2-s-\ell} \sum_{\rho_{n} \geq 1}\left\langle f, v_{n}\right\rangle u_{n}\left(x_{0}\right) \int_{0}^{R} r^{-s} J_{N / 2+s-\ell}(\lambda r) \times \\
& \quad \times\left\{\frac{J_{N / 2-\ell-1}\left(\mu_{n} r\right)}{\mu_{n}^{N / 2-\ell-1}}-\frac{J_{N / 2-\ell-1}\left(\rho_{n} r\right)}{\rho_{n}^{N / 2-\ell-1}}\right\} \mathrm{d} r .
\end{aligned}
$$

In case $s=0, \sigma_{\lambda, 1}^{s}\left(f, x_{0}\right)$ has to be substituted by

$$
\sigma_{\lambda, 1}^{s}\left(f, x_{0}\right)-\frac{1}{2} \sum_{\lambda=\rho_{n}}\left\langle f, v_{n}\right\rangle u_{n}\left(x_{0}\right)
$$

Proof.- Define the function

$$
v^{\lambda}(r):= \begin{cases}\frac{\Gamma(s+1) 2^{s}}{(2 \pi)^{N / 2}} \lambda^{N / 2-s} \frac{J_{N / 2+s}(\lambda r)}{r^{N / 2+s}} & 0 \leq r \leq R \\ 0 & R<r\end{cases}
$$

and expand the function $v^{\lambda}\left(\left|x-x_{0}\right|\right)$ by the Riesz basis $\left\{v_{n}\right\}$. The coefficients have the form

$$
\begin{aligned}
& \left\langle u_{n}, v^{\lambda}\left(\left|x-x_{0}\right|\right)\right\rangle= \\
& =\int_{|z| \leq R} u_{n}(x+z) v^{\lambda}(|z|) \mathrm{d} z \\
& =\Gamma(s+1) 2^{s}(2 \pi)^{-N / 2} \lambda^{N / 2-s} \int_{0}^{R} r^{N / 2-s-1} J_{N / 2+s}(\lambda r) \int_{\theta} u_{n}\left(x_{0}+r \theta\right) \mathrm{d} \theta \mathrm{d} r
\end{aligned}
$$


M. Horváth

$$
\begin{aligned}
= & \Gamma(s+1) 2^{s} \lambda^{N / 2-s} u_{n}\left(x_{0}\right) \int_{0}^{R} r^{-N / 2-s+1} J_{N / 2+s}(\lambda r) \times \\
& \times\left(\frac{r}{\mu_{n}}\right)^{N / 2-1} J_{N / 2-1}\left(\mu_{n} r\right) \mathrm{d} r \\
= & \Gamma(s+1) 2^{s} \lambda^{N / 2-s} u_{n}\left(x_{0}\right)\left\{-\lambda^{-1} R^{-s} J_{N / 2+s-1}(\lambda R) \frac{J_{N / 2-1}\left(\mu_{n} R\right)}{\mu_{n}^{N / 2-1}}+\right. \\
& \left.+\lambda^{-1} \int_{0}^{R} r^{-s} J_{N / 2+s-1}(\lambda r) \frac{J_{N / 2-2}\left(\mu_{n} r\right)}{\mu_{n}^{N / 2-2}} \mathrm{~d} r\right\} \\
= & \Gamma(s+1) 2^{s} \lambda^{N / 2-s} u_{n}\left(x_{0}\right)\left\{-\lambda^{-1} R^{-s} J_{N / 2+s-1}(\lambda R) \frac{J_{N / 2-1}\left(\mu_{n} R\right)}{\mu_{n}^{N / 2-1}}-\cdots\right. \\
& \ldots-\lambda^{-\ell} R^{-s} J_{N / 2+s-\ell}(\lambda R) \frac{J_{N / 2-\ell}\left(\mu_{n} R\right)}{\mu_{n}^{N / 2-\ell}+} \\
& \left.+\lambda^{-\ell} \int_{0}^{R} r^{-s} J_{N / 2+s-\ell}(\lambda r) \frac{J_{N / 2-\ell-1}\left(\mu_{n} r\right)}{\mu_{n}^{N / 2-\ell-1}} \mathrm{~d} r\right\} .
\end{aligned}
$$

Since $f=\sum\left\langle f, v_{n}\right\rangle u_{n}$ and $v^{\lambda}=\sum\left\langle v^{\lambda}, u_{n}\right\rangle v_{n}$ are convergent in $L_{2}$, we get from here that

$$
\begin{aligned}
\left\langle f, v^{\lambda}\right\rangle= & \sum\left\langle f, v_{n}\right\rangle\left\langle u_{n}, v^{\lambda}\right\rangle \\
=- & \Gamma(s+1) 2^{s} \sum_{k=0}^{\ell-1} \lambda^{N / 2-s-k-1} J_{N / 2+s-k-1}(\lambda R) \times \\
& \times R^{-N / 2+k-s} \frac{\omega_{N}}{(2 \pi)^{N / 2}} \mathrm{D}^{k} F(R)+ \\
+ & \Gamma(s+1) 2^{s} \lambda^{N / 2-s-\ell} \sum\left\langle f, v_{n}\right\rangle u_{n}\left(x_{0}\right) \times \\
& \times \int_{0}^{R} r^{-s} J_{N / 2+s-\ell}(\lambda r) \frac{J_{N / 2-\ell-1}\left(\mu_{n} r\right)}{\mu_{n}^{N / 2-\ell-1}} \mathrm{~d} r
\end{aligned}
$$

by Lemma $6 \mathrm{~b}$ ). For the indices $\rho_{n} \geq 1$, we write

$$
\int_{0}^{R} r^{-s} J_{N / 2+s-\ell}(\lambda r) J_{N / 2-\ell-1}\left(\mu_{n} r\right) \mu_{n}^{\ell+1-N / 2} \mathrm{~d} r=
$$


Local uniform convergence of the Riesz means of Laplace and Dirac expansions

$$
\begin{aligned}
= & \left(\int_{0}^{\infty}-\int_{R}^{\infty}\right) r^{-s} J_{N / 2+s-\ell}(\lambda r) J_{N / 2-\ell-1}\left(\rho_{n} r\right) \rho_{n}^{\ell+1-N / 2} \mathrm{~d} r+ \\
& +\int_{0}^{R} r^{-s} J_{N / 2+s-\ell}(\lambda r)\left\{\frac{J_{N / 2-\ell-1}\left(\mu_{n} r\right)}{\mu_{n}^{N / 2-\ell-1}}-\frac{J_{N / 2-\ell-1}\left(\rho_{n} r\right)}{\rho_{n}^{N / 2-\ell-1}}\right\} \mathrm{d} r .
\end{aligned}
$$

Here in case $s>0$, we have by $[3, \S 7.14 .2,7.7 .4]$

$$
\begin{aligned}
\int_{0}^{\infty} r^{-s} J_{N / 2+s-\ell}(\lambda r) J_{N / 2-\ell-1}\left(\rho_{n} r\right) \rho_{n}^{\ell+1-N / 2} \mathrm{~d} r= \\
= \begin{cases}0 & \text { if } \lambda \leq \rho_{n} \\
\frac{\lambda^{\ell-s-N / 2}}{\Gamma(s+1) 2^{s}}\left(\lambda^{2}-\rho_{n}^{2}\right)^{s} & \text { if } \lambda>\rho_{n} .\end{cases}
\end{aligned}
$$

In case $s=0$, we obtain by $[3, \S 13.41]$ that

$$
\int_{0}^{\infty} J_{N / 2-\ell}(\lambda r) \frac{J_{N / 2-\ell-1}\left(\rho_{n} r\right)}{\rho_{n}^{N / 2-\ell-1}} \mathrm{~d} r= \begin{cases}0 & \text { if } \lambda<\rho_{n} \\ \frac{1}{2} \lambda^{\ell-N / 2} & \text { if } \lambda=\rho_{n} \\ \lambda^{\ell-N / 2} & \text { if } \lambda>\rho_{n}\end{cases}
$$

Thus in case $s>0$,

$$
\begin{aligned}
& \left\langle f, v^{\lambda}\right\rangle= \\
& =-\frac{\Gamma(s+1) 2^{s}}{(2 \pi)^{N / 2}} \omega_{N} \sum_{k=0}^{\ell-1} \lambda^{N / 2-s-k-1} \frac{J_{N / 2+s-k-1}(\lambda R)}{R^{N / 2+s-k}} \mathrm{D}^{k} F(R)+ \\
& \quad+\Gamma(s+1) 2^{s} \lambda^{N / 2-s-\ell} \sum_{\rho_{n}<1}\left\langle f, v_{n}\right\rangle u_{n}\left(x_{0}\right) \times \\
& \quad \times \int_{0}^{R} r^{-s} J_{N / 2+s-\ell}(\lambda r) J_{N / 2-\ell-1}\left(\mu_{n} r\right) \mu_{n}^{\ell+1-N / 2} \mathrm{~d} r+ \\
& +\sigma_{\lambda}^{s}\left(f, x_{0}\right)-\sigma_{\lambda, 1}^{s}\left(f, x_{0}\right)-\Gamma(s+1) 2^{s} \lambda^{N / 2-s-\ell} \sum_{\rho_{n} \geq 1}\left\langle f, v_{n}\right\rangle u_{n}\left(x_{0}\right) \times \\
& \quad \times \int_{R}^{\infty} r^{-s} J_{N / 2+s-\ell}(\lambda r) \frac{J_{N / 2-\ell-1}\left(\rho_{n} r\right)}{\rho_{n}^{N / 2-\ell-1} \mathrm{~d} r+} \\
& \quad+\Gamma(s+1) 2^{s} \lambda^{N / 2-s-\ell} \sum_{\rho_{n} \geq 1}\left\langle f, v_{n}\right\rangle u_{n}\left(x_{0}\right) \times \\
& \quad \times \int_{0}^{R} r^{-s} J_{N / 2+s-\ell}(\lambda r)\left\{\frac{J_{N / 2-\ell-1}\left(\mu_{n} r\right)}{\mu_{n}^{N / 2-\ell-1}}-\frac{J_{N / 2-\ell-1}\left(\rho_{n} r\right)}{\rho_{n}^{N / 2-\ell-1}}\right\} \mathrm{d} r
\end{aligned}
$$




\section{Horváth}

and for $s=0$ the same formula holds with

$$
\sigma_{\lambda, 1}^{s}\left(f, x_{0}\right)-\frac{1}{2} \sum_{\lambda=\rho_{n}}\left\langle f, v_{n}\right\rangle u_{n}\left(x_{0}\right) \quad \text { instead of } \sigma_{\lambda, 1}^{s}\left(f, x_{0}\right) .
$$

On the other hand, iterated integration by parts gives

$$
\begin{aligned}
& \left\langle f, v^{\lambda}\right\rangle= \\
& =\frac{\Gamma(s+1) 2^{s}}{(2 \pi)^{N / 2}} \lambda^{N / 2-s} \int_{0}^{R} r^{N / 2-1-s} J_{N / 2+s}(\lambda r) \int_{\theta} f\left(x_{0}+r \theta\right) \mathrm{d} \theta \mathrm{d} r \\
& =\frac{\Gamma(s+1) 2^{s}}{(2 \pi)^{N / 2}} \omega_{N} \lambda^{N / 2-s} \int_{0}^{R} F(r) \frac{J_{N / 2+s}(\lambda r)}{r^{N / 2+s}} \mathrm{~d} r \\
& =\frac{\Gamma(s+1) 2^{s}}{(2 \pi)^{N / 2}} \omega_{N} \lambda^{N / 2-s}\left(\lambda^{-\ell} \int_{0}^{R} \mathrm{D}^{\ell} F(r) \frac{J_{N / 2+s-\ell}(\lambda r)}{r^{N / 2+s-\ell}} \mathrm{d} r+\right. \\
& \left.\quad-\sum_{k=0}^{\ell-1} \lambda^{-k-1} \mathrm{D}^{k} F(R) \frac{J_{N / 2+s-k-1}(\lambda R)}{R^{N / 2+s-k}}\right) .
\end{aligned}
$$

Comparing the two expressions for $\left\langle f, v^{\lambda}\right\rangle$ proves Lemma 8.

LEMMA 9

a) If $\lambda \rho \leq 4 \pi$, then

$$
\left|\sigma_{\lambda}^{s}\left(f, x_{0}\right)\right| \leq c \lambda^{N / 2-\alpha}\|f\|_{H_{2}^{\alpha}} .
$$

b) If $\lambda \rho \geq 4 \pi$, then

$$
\begin{gathered}
\left|\sigma_{\lambda, 1}^{s}\left(f, x_{0}\right)\right| \leq c\|f\|_{H_{2}^{\alpha}} . \\
\text { In case } s=0, \quad\left|\sum_{\lambda=\rho_{n}}\left\langle f, v_{n}\right\rangle u_{n}\left(x_{0}\right)\right| \leq c\|f\|_{H_{2}^{\alpha}}, \\
\mid \sum_{\rho_{n} \geq 1}\left\langle f, v_{n}\right\rangle u_{n}\left(x_{0}\right) \lambda^{N / 2-s-\ell} \int_{R}^{\infty} r^{-s} J_{N / 2+s-\ell}(\lambda r) \times \\
\times J_{N / 2-\ell-1}\left(\rho_{n} r\right) \rho_{n}^{\ell+1-N / 2} \mathrm{~d} r \mid \leq c\|f\|_{H_{2}^{\alpha}}
\end{gathered}
$$


Local uniform convergence of the Riesz means of Laplace and Dirac expansions

$$
\begin{aligned}
\lambda^{N / 2-s-\ell} \mid \sum_{\rho_{n}<1}\left\langle f, v_{n}\right\rangle & u_{n}\left(x_{0}\right) \int_{0}^{R} r^{-s} J_{N / 2+s-\ell}(\lambda r) \times \\
& \times J_{N / 2-\ell-1}\left(\mu_{n} r\right) \mu_{n}^{\ell+1-N / 2} \mathrm{~d} r \mid \leq c\|f\|_{H_{2}^{\alpha}}
\end{aligned}
$$

The constants may depend on $R$, i.e., on $\operatorname{dist}\left(x_{0}, \partial \Omega\right)$.

Proof. - Define $m^{*}$ by the property $\lambda \leq 2^{m^{*}}<2 \lambda$. We have

$$
\begin{aligned}
\left|\sigma_{\lambda, 1}^{s}\left(f, x_{0}\right)\right| & \leq \sum_{\rho_{n}<1}\left|\left\langle f, v_{n}\right\rangle u_{n}\left(x_{0}\right)\right| \\
& \leq\left(\sum\left|\left\langle f, v_{n}\right\rangle\right|^{2}\right)^{1 / 2}\left(\sum_{\rho_{n}<1}\left|u_{n}\left(x_{0}\right)\right|^{2}\right)^{1 / 2} \\
& \leq c\|f\|_{L_{2}} \leq c\|f\|_{H_{2}^{\alpha}}
\end{aligned}
$$

which shows (25). Now for $m \leq m^{*}-2$

$$
\begin{aligned}
& \left|\sum_{2^{m} \leq \rho_{n}<2^{m+1}}\left\langle f, v_{n}\right\rangle u_{n}\left(x_{0}\right)\left(1-\rho_{n}^{2} \lambda^{-2}\right)^{s}\right| \leq \\
& \leq c\left(\sum\left|\left\langle f, v_{n}\right\rangle\right|^{2}\right)^{1 / 2}\left(\sum\left|u_{n}\left(x_{0}\right)\right|^{2}\right)^{1 / 2} \leq c\|f\|_{H_{2}^{\alpha}} 2^{m(N / 2-\alpha)}
\end{aligned}
$$

and analogously

$$
\left|\sum_{2^{m^{*}-1} \leq \rho_{n}<\lambda}\left\langle f, v_{n}\right\rangle u_{n}\left(x_{0}\right)\left(1-\rho_{n}^{2} \lambda^{-2}\right)^{s}\right| \leq c\|f\|_{H_{2}^{\alpha} 2^{m^{*}(N / 2-\alpha)}}
$$

Summing up for $m \leq m^{*}-2$ the estimate (24) follows. In case $s=0$, we have

$$
\begin{aligned}
&\left|\sum_{\lambda=\rho_{n}}\left\langle f, v_{n}\right\rangle u_{n}\left(x_{0}\right)\right| \leq\left(\sum\left|\left\langle f, v_{n}\right\rangle\right|^{2}\right)^{1 / 2}\left(\sum\left|u_{n}\left(x_{0}\right)\right|^{2}\right)^{1 / 2} \\
& \leq c\|f\|_{H_{2}^{\alpha} \lambda^{(N-1) / 2-\alpha}=c\|f\|_{H_{2}^{\alpha}}} \\
&-681-
\end{aligned}
$$


so $(26)$ is proved. To show (27), we use the estimate $[23, \S 18.10]$

$$
\begin{aligned}
\left|\int_{R}^{\infty} r^{-s} J_{N / 2+s-\ell}(\lambda r) J_{N / 2-\ell-1}\left(\rho_{n} r\right) \mathrm{d} r\right| \leq \\
\leq \frac{c}{\left(\lambda \rho_{n}\right)^{1 / 2}\left(1+\left|\lambda-\rho_{n}\right|\right)}, \quad \rho_{n} \geq 1 .
\end{aligned}
$$

Applying this in (27) we have

$$
\begin{aligned}
& \left|\sum_{\rho_{n} \geq 3 / 2 \lambda}\right| \leq c \lambda^{(N-1) / 2-s-\ell} \sum_{\rho_{n} \geq 3 / 2 \lambda}\left|\left\langle f, v_{n}\right\rangle u_{n}\left(x_{0}\right)\right| \rho_{n}^{\ell-(N+1) / 2} \\
& \leq c \lambda^{\chi} \sum_{m=0}^{\infty}\left(\sum_{2^{m} \lambda \leq \rho_{n} \leq 2^{m+1} \lambda}\left|\left\langle f, v_{n}\right\rangle\right|^{2} \rho_{n}^{2 \alpha}\right)^{1 / 2} \times \\
& \times\left(\sum_{2^{m} \lambda \leq \rho_{n} \leq 2^{m+1} \lambda} \frac{\left|u_{n}\left(x_{0}\right)\right|^{2}}{\rho_{n}^{N+1+2 \chi}}\right)^{1 / 2} \\
& \leq c\|f\|_{H_{2}^{\alpha}} \sum_{m=0}^{\infty}\left(\sum \frac{\left|u_{n}\left(x_{0}\right)\right|^{2}}{\rho_{n}^{N+1}}\right)^{1 / 2} \leq c\|f\|_{H_{2}^{\alpha}} \\
& \left|\sum_{\left|\rho_{n}-\lambda\right| \leq \lambda / 2}\right| \leq c \lambda^{\chi+1 / 2} \sum_{\left|\rho_{n}-\lambda\right| \leq \lambda / 2} \frac{\left|\left\langle f, v_{n}\right\rangle u_{n}\left(x_{0}\right)\right|}{\lambda\left(1+\left|\lambda-\rho_{n}\right|\right)} \lambda^{\ell+1-N / 2} \\
& \leq c \lambda^{-s}\left(\sum_{\left|\rho_{n}-\lambda\right| \leq \lambda / 2}\left|\left\langle f, v_{n}\right\rangle\right|^{2} \rho_{n}^{2 \alpha}\right)^{1 / 2} \times \\
& \times\left(\sum_{\left|\rho_{n}-\lambda\right| \leq 1}\left|u_{n}\left(x_{0}\right)\right|^{2} \rho_{n}^{-2 \alpha}+\right. \\
& \left.+\sum_{m=0}^{m^{*}} \sum_{2^{m} \leq\left|\rho_{n}-\lambda\right| \leq 2^{m+1}}\left|u_{n}\left(x_{0}\right)\right|^{2} \rho_{n}^{-2 \alpha} 2^{-2 m}\right)^{1 / 2} \\
& \leq c \lambda^{-s}\|f\|_{H_{2}^{\alpha}}\left(\lambda^{N-1-2 \alpha}+\sum_{m=0}^{m^{*}} 2^{-2 m} 2^{m} \lambda^{N-1-2 \alpha}\right)^{1 / 2} \\
& \leq c\|f\|_{H_{2}^{\alpha}} \text {; }
\end{aligned}
$$


Local uniform convergence of the Riesz means of Laplace and Dirac expansions

$$
\begin{aligned}
\left|\sum_{1 \leq \rho_{n} \leq \lambda / 2}\right| \leq c \lambda^{\chi+1 / 2} \sum_{1 \leq \rho_{n} \leq \lambda / 2}\left|\left\langle f, v_{n}\right\rangle u_{n}\left(x_{0}\right)\right| \lambda^{-3 / 2} \rho_{n}^{\ell-(N-1) / 2} \\
\leq c \lambda^{\chi-1} \sum_{m=0}^{m^{*}}\left(\sum_{2^{m} \leq \rho_{n} \leq 2^{m+1}}\left|\left\langle f, v_{n}\right\rangle\right|^{2} \rho_{n}^{2 \alpha}\right)^{1 / 2} \times \\
\\
\times\left(\sum_{2^{m} \leq \rho_{n} \leq 2^{m+1}}\left|u_{n}\left(x_{0}\right)\right|^{2} \rho_{n}^{2 \ell+1-N-2 \alpha}\right)^{1 / 2} \\
\leq c \lambda^{\chi-1}\|f\|_{H_{2}^{\alpha}} \sum_{m=0}^{m^{*}} 2^{m(1 / 2-\chi)} .
\end{aligned}
$$

Now if $1 / 2<\chi$ then we can continue this estimate (leaving the norm of $f$ ) by $\leq c \lambda^{-1} \leq c$; if $1 / 2=\chi$ then by $\leq c \lambda^{-1 / 2} \ln \lambda \leq c$; finally if $1 / 2>\chi$ then by $\leq c \lambda^{\chi^{-1}} \lambda^{1 / 2-\chi} \leq c$. So the statement (27) is proved; we investigate (28). Take the expansion

$$
\begin{gathered}
\frac{J_{N / 2-\ell-1}\left(\mu_{n} r\right)}{\left(\mu_{n} r\right)^{N / 2-\ell-1}}=\sum_{m=0}^{\infty} c_{m}\left(\mu_{n} r\right)^{2 m}, \\
c_{m}=\frac{(-1)^{m}}{2^{2 m+N / 2-\ell-1} m ! \Gamma(N / 2-\ell+m)} .
\end{gathered}
$$

Applying this we get

$$
\begin{aligned}
& \int_{0}^{R} r^{-s} J_{N / 2+s-\ell}(\lambda r) J_{N / 2-\ell-1}\left(\mu_{n} r\right) \mu_{n}^{\ell+1-N / 2} \mathrm{~d} r= \\
& =c_{0} \int_{0}^{R} r^{\chi-1 / 2} J_{N / 2+s-\ell}(\lambda r) \mathrm{d} r+ \\
& \quad+\sum_{m=1}^{\infty} c_{m} \mu_{n}^{2 m} \int_{0}^{R} r^{\chi-1 / 2+2 m} J_{N / 2+s-\ell}(\lambda r) \mathrm{d} r .
\end{aligned}
$$

We shall prove that

$$
\left|\int_{0}^{R} r^{\chi-1 / 2} J_{N / 2+s-\ell}(\lambda r) \mathrm{d} r\right| \leq c \lambda^{-\chi-1 / 2} .
$$


Indeed :

$$
\left|\int_{0}^{R / \lambda}\right| \leq c \int_{0}^{R / \lambda} r^{\chi-1 / 2} \mathrm{~d} r \leq c \lambda^{-\chi-1 / 2}
$$

for $r \geq R / \lambda$, we use the formula

$$
\begin{aligned}
J_{\nu}(x)=\left(\frac{2}{\pi x}\right)^{1 / 2} & \left\{\cos \left(x-\frac{\pi}{2}\left(\nu+\frac{1}{2}\right)\right)+\right. \\
& \left.+\frac{4 \nu^{2}-1}{8} \frac{\cos \left(x-\frac{\pi}{2}\left(\nu-\frac{1}{2}\right)\right)}{x}\right\}+\mathrm{O}\left(x^{-5 / 2}\right) .
\end{aligned}
$$

For the remainder term

$$
\int_{R / \lambda}^{R} r^{\chi-1 / 2}(\lambda r)^{-5 / 2} \mathrm{~d} r=\lambda^{-5 / 2} \int_{R / \lambda}^{R} r^{\chi-3} \mathrm{~d} r \leq c \lambda^{-1 / 2-\chi}
$$

for the second main term, we get by integration by parts

$$
\lambda^{-3 / 2}\left|\int_{R / \lambda}^{R} r^{\chi-2} \sin (\lambda r+\gamma) \mathrm{d} r\right| \leq c \lambda^{-1 / 2-\chi}
$$

and analogously for the first main term twofold integration gives

$$
\lambda^{-1 / 2}\left|\int_{R / \lambda}^{R} r^{\chi-1} \cos (\lambda r+\gamma) \mathrm{d} r\right| \leq c \lambda^{-1 / 2-\chi}
$$

which proves (31). Similarly for $m \geq 1$,

$$
\left|\int_{0}^{R} r^{\chi-1 / 2+2 m} J_{N / 2+s-\ell}(\lambda r) \mathrm{d} r\right| \leq c \lambda^{-3 / 2} R^{2 m}
$$

where $c$ is independent of $m$. Indeed, for the remainder of (21)

$$
\int_{0}^{R}{ }^{\chi-1 / 2+2 m}(\lambda r)^{-3 / 2} \mathrm{~d} r=\lambda^{-3 / 2} \int_{0}^{R} r^{\chi-2+2 m} \mathrm{~d} r \leq c \lambda^{-3 / 2} R^{2 m}
$$

and for the main term we integrate by parts to obtain

$$
\lambda^{-1 / 2}\left|\int_{0}^{R} r^{\chi-1+2 m} \cos (\lambda r+\gamma) \mathrm{d} r\right| \leq c \lambda^{-3 / 2} R^{2 m} .
$$


Local uniform convergence of the Riesz means of Laplace and Dirac expansions

Thus we have by $(30)$

$$
\begin{aligned}
\lambda^{\chi+1 / 2}\left|\sum_{\rho_{n}<1}\left\langle f, v_{n}\right\rangle u_{n}\left(x_{0}\right) \int_{0}^{R} r^{-s} J_{N / 2+s-\ell}(\lambda r) \frac{J_{N / 2-\ell-1}\left(\mu_{n} r\right)}{\mu_{n}^{N / 2-\ell-1}} \mathrm{~d} r\right| \leq \\
\quad \leq c \lambda^{\chi+1 / 2} \sum_{\rho_{n}<1}\left|\left\langle f, v_{n}\right\rangle u_{n}\left(x_{0}\right)\right|\left(\left|c_{0}\right| \lambda^{-\chi-1 / 2}+\sum_{m=1}^{\infty}\left|c_{m}\right|\left|\mu_{n}\right|^{2 m} \frac{R^{2 m}}{\lambda^{3 / 2}}\right) \\
\quad \leq c \sum_{\rho_{n}<1}\left|\left\langle f, v_{n}\right\rangle u_{n}\left(x_{0}\right)\right|\left(1+\sum_{m=1}^{\infty}\left(\frac{|R| \mu_{n}}{2}\right)^{2 m}((m-1) !)^{-2}\right) \\
\left.\quad \leq c \sum_{\rho_{n}<1}\left|\left\langle f, v_{n}\right\rangle u_{n}\left(x_{0}\right)\right| e^{2\left|\nu_{n}\right| R}\right) \\
\quad \leq c\left(\sum_{\rho_{n}<1}\left|\left\langle f, v_{n}\right\rangle\right|^{2}\right)^{1 / 2}\left(\sum_{\rho_{n}<1}\left|u_{n}\left(x_{0}\right)\right|^{2} e^{4\left|\nu_{n}\right| R}\right) \leq c\|f\|_{H_{2}^{\alpha} .}
\end{aligned}
$$

Lemma 9 is proved.

\section{Completing the proof of Lemma 1}

In this section we prove two further lemmas but first we show how to deduce then Lemma 1.

LEMMA $10 .-$ Let $0 \leq s<1 / 2,0<2 \varepsilon<1 / 2-s$ and $\lambda, \rho_{n} \geq 1$. Then

$$
\begin{aligned}
& \left|\int_{0}^{R} r^{-s} J_{1+s}(\lambda r)\left(J_{0}\left(\mu_{n} r\right)-J_{0}\left(\rho_{n} r\right)\right) \mathrm{d} r\right| \leq \\
& \quad \leq c e^{\left|\nu_{n}\right| R}\left(1+\left|\lambda-\rho_{n}\right|\right)^{-1 / 2-\varepsilon} \lambda^{s-1 / 2+\varepsilon} \rho_{n}^{-s-1 / 2-\varepsilon} .
\end{aligned}
$$

Lemma11. - Let $0 \leq s<1 / 2,0<2 \varepsilon<1 / 2-s$ and $\lambda, \rho_{n} \geq 1$. Then

$$
\begin{gathered}
\left|\int_{0}^{R} r^{-s} J_{3 / 2+s}(\lambda r)\left(\mu_{n}^{-1 / 2} J_{1 / 2}\left(\mu_{n} r\right)-\rho_{n}^{-1 / 2} J_{1 / 2}\left(\rho_{n} r\right)\right) \mathrm{d} r\right| \leq \\
\leq c e^{\left|\nu_{n}\right| R}\left(1+\left|\lambda-\rho_{n}\right|\right)^{-1 / 2-\varepsilon} \lambda^{s-1+\varepsilon} \rho_{n}^{-s-1 / 2-\varepsilon} . \\
-685-
\end{gathered}
$$




\section{Horváth}

\section{Proof of Lemma 1}

(through Lemmas 10 and 11) If $\lambda \rho \leq 4 \pi,(24)$ gives the desired estimate. If $\lambda \rho \geq 4 \pi$, choose $R>0$ such that

$$
\sum_{\left|\mu-\rho_{n}\right| \leq 1}\left|u_{n}\left(x_{0}\right)\right|^{2} e^{4\left|\nu_{n}\right| R} \leq c \mu^{N-1}
$$

holds, further $R \geq c \operatorname{dist}(x, \partial \Omega)$ with an absolute constant $c>0$ and

$$
\cos (\lambda R+\gamma)=0, \quad \gamma=-\frac{\pi}{2}\left(\frac{N+1}{2}+1+s-\ell\right) .
$$

This last condition means that we introduce $R+\mathrm{O}(1 / \lambda)$ instead of $R$. Since $\lambda \rho \geq 4 \pi$, this dependence of $R$ on $\lambda$ does not disturb our estimates. Now consider the expression (23) for $\sigma_{\lambda}^{s}\left(f, x_{0}\right)$. For the first three (resp. four in case $s=0$ ) terms the needed estimates are proved in Lemma 9 and the fourth (resp. fifth) component is estimated in Lemma 7. So it remains the last member in (23). The expression $s+\chi-1 / 2=N / 2-\ell-1$ is the half of an integer and belongs to the interval $(-1 / 2,1)$; hence it equals to 0 or $1 / 2$. Consider separately these cases.

A) $\chi=1 / 2-s$

Then we have to prove that

$$
\begin{gathered}
\lambda^{\chi+1 / 2}\left|\sum_{\rho_{n} \geq 1}\left\langle f, v_{n}\right\rangle u_{n}\left(x_{0}\right) \int_{0}^{R} r^{-s} J_{1+s}(\lambda r)\left(J_{0}\left(\mu_{n} r\right)-J_{0}\left(\rho_{n} r\right)\right) \mathrm{d} r\right| \leq \\
\leq c \rho^{\alpha-N / 2-\varepsilon}\|f\|_{H_{2}^{\alpha}} .
\end{gathered}
$$

In fact the stronger estimate $\leq c\|f\|$ holds, since

$$
\begin{aligned}
& \lambda^{1-s}\left|\sum_{\rho_{n} \geq 3 / 2 \lambda}\right| \leq \\
& \leq \lambda^{1-s} \sum_{k=-1}^{\infty}\left(\sum_{3 \cdot 2^{k} \lambda \leq \rho_{n} \leq 3 \cdot 2^{k+1} \lambda}\left|\left\langle f, v_{n}\right\rangle\right|^{2} \rho_{n}^{2 \alpha}\right)^{1 / 2} \times \\
& \quad \times\left(\sum_{3 \cdot 2^{k} \lambda \leq \rho_{n} \leq 3 \cdot 2^{k+1} \lambda} \frac{\left|u_{n}\left(x_{0}\right)\right|^{2}}{\rho_{n}^{2 \alpha}} \frac{e^{2\left|\nu_{n}\right| R}}{\rho_{n}^{1+2 \varepsilon}} \frac{\lambda^{2 s-1+2 \varepsilon}}{\rho_{n}^{2 s+1+2 \varepsilon}}\right)^{1 / 2} \\
& \leq c\|f\|_{H_{2}^{\alpha}} \sum_{k=-1}^{\infty}\left(\lambda^{-2 \varepsilon} 2^{-k(1+4 \varepsilon)}\right)^{1 / 2} \leq c\|f\|_{H_{2}^{\alpha}} ;
\end{aligned}
$$


Local uniform convergence of the Riesz means of Laplace and Dirac expansions

$$
\begin{aligned}
& \lambda^{1-s}\left|\sum_{1 \leq \rho_{n} \leq \lambda / 2}\right| \leq \lambda^{1-s} \sum_{k=0}^{k^{*}}\left(\sum_{2^{k} \leq \rho_{n} \leq 2^{k+1}}\left|\left\langle f, v_{n}\right\rangle\right|^{2} \rho_{n}^{2 \alpha}\right)^{1 / 2} \times \\
& \times\left(\sum_{2^{k} \leq \rho_{n} \leq 2^{k+1}}\left|u_{n}\left(x_{0}\right)\right|^{2} \lambda^{2 s-2} \rho_{n}^{-(2 \alpha+2 s+1+2 \varepsilon)}\right)^{1 / 2} \\
& \leq c\|f\|_{H_{2}^{\alpha}} \sum_{k=0}^{k^{*}} 2^{-k \varepsilon} \leq c\|f\|_{H_{2}^{\alpha}} \\
& \lambda^{1-s}\left|\sum_{\left|\lambda-\rho_{n}\right| \leq \lambda / 2}\right| \leq \\
& \leq \lambda^{1-s}\left(\sum_{\left|\lambda-\rho_{n}\right| \leq \lambda / 2}\left|\left\langle f, v_{n}\right\rangle\right|^{2} \rho_{n}^{2 \alpha}\right)^{1 / 2}\left(\sum_{\left|\lambda-\rho_{n}\right| \leq \lambda / 2}\left|u_{n}\left(x_{0}\right)\right|^{2} \times\right. \\
& \left.\times e^{2\left|\nu_{n}\right| R}\left(1+\left|\lambda-\rho_{n}\right|\right)^{-1-2 \varepsilon} \lambda^{2 s-1+2 \varepsilon} \rho_{n}^{-(2 \alpha+2 s+1+2 \varepsilon)}\right)^{1 / 2} \\
& \leq c\|f\|_{H_{2}^{\alpha}} \lambda^{-\alpha-s}\left(\sum_{\left|\lambda-\rho_{n}\right| \leq 1}\left|u_{n}\left(x_{0}\right)\right|^{2} e^{2\left|\nu_{n}\right| R}+\right. \\
& \left.+\sum_{k=0}^{k^{*}} 2^{-k(1+2 \varepsilon)} \sum_{2^{k} \leq\left|\lambda-\rho_{n}\right| \leq 2^{k+1}}\left|u_{n}\left(x_{0}\right)\right|^{2} e^{2\left|\nu_{n}\right| R}\right)^{1 / 2} \\
& \leq c \lambda^{-\alpha-s}\|f\|_{H_{2}^{\alpha}} \lambda^{(N-1) / 2}\left(1+\sum_{k=0}^{k^{*}} 2^{-2 k \varepsilon}\right)^{1 / 2} \leq c\|f\|_{H_{2}^{\alpha}}
\end{aligned}
$$

as we asserded.

B) $\chi=1-s$

As in A), we show that the stronger estimate holds, namely

$$
\begin{aligned}
\lambda^{3 / 2-} & \mid \sum_{\rho_{n} \geq 1}\left\langle f, v_{n}\right\rangle u_{n}\left(x_{0}\right) \int_{0}^{R} r^{-s} J_{3 / 2+s}(\lambda r) \times \\
& \times\left(\mu_{n}^{-1 / 2} J_{1 / 2}\left(\mu_{n} r\right)-\rho_{n}^{-1 / 2} J_{1 / 2}\left(\rho_{n}\right)\right) \mathrm{d} r \mid \leq c\|f\|_{H_{2}^{\alpha}} .
\end{aligned}
$$




\section{Horváth}

Indeed,

$$
\begin{aligned}
& \lambda^{3 / 2-s}\left|\sum_{\rho_{n} \geq 3 / 2 \lambda}\right| \leq \\
& \leq c\|f\|_{H_{2}^{\alpha}} \sum_{k=-1}^{\infty}\left(\lambda^{3-2 s} \sum_{3.2^{k} \lambda \leq \rho_{n} \leq 3.2^{k+1} \lambda} \frac{\left|u_{n}\left(x_{0}\right)\right|^{2} \lambda^{2 s-2+2 \varepsilon}}{\left(2^{k} \lambda\right)^{2 \alpha+2+2 s+4 \varepsilon}} e^{2\left|\nu_{n}\right| R}\right)^{1 / 2} \\
& \leq c\|f\|_{H_{2}^{\alpha} \lambda^{-\varepsilon}} \sum_{k=-1}^{\infty} 2^{-k(1 / 2+2 \varepsilon)} \leq c\|f\|_{H_{2}^{\alpha}} \\
& \lambda^{3 / 2-s}\left|\sum_{1 \leq \rho_{n} \leq \lambda / 2}\right| \leq \\
& \leq c\|f\|_{H_{2}^{\alpha}} \sum_{k=0}^{k^{*}}\left(\sum_{2^{k} \leq \rho_{n} \leq 2^{k+1}} 2^{-k(2 \alpha+2 s+1+2 \varepsilon)}\left|u_{n}\left(x_{0}\right)\right|^{2} 2^{2\left|\nu_{n}\right| R}\right)^{1 / 2} \\
& \leq c\|f\|_{H_{2}^{\alpha}} \sum_{k=0}^{k^{*}} 2^{-k \varepsilon} \leq c\|f\|_{H_{2}^{\alpha}} \text {; } \\
& \lambda^{3 / 2-s}\left|\sum_{\left|\lambda-\rho_{n}\right| \leq \lambda / 2}\right| \leq \\
& \leq c \lambda^{-s-\alpha}\|f\|_{H_{2}^{\alpha}}\left(\sum_{\left|\lambda-\rho_{n}\right| \leq \lambda / 2}\left|u_{n}\left(x_{0}\right)\right|^{2} e^{2\left|\nu_{n}\right| R}\left(1+\left|\lambda-\rho_{n}\right|\right)^{-1-2 \varepsilon}\right)^{1 / 2} \\
& \leq c\|f\|_{H_{2}^{\alpha} \lambda^{-s-\alpha}}\left(\sum_{\left|\lambda-\rho_{n}\right| \leq 1}\left|u_{n}\left(x_{0}\right)\right|^{2} e^{2\left|\nu_{n}\right| R}+\right. \\
& \left.+\sum_{k=0}^{k^{*}} 2^{-k(1+2 \varepsilon)} \sum_{2^{k} \leq\left|\lambda-\rho_{n}\right| \leq 2^{k+1}}\left|u_{n}\left(x_{0}\right)\right|^{2} e^{2\left|\nu_{n}\right| R}\right)^{1 / 2} \\
& \leq c\|f\|_{H_{2}^{\alpha}}\left(1+\sum_{k=k_{0}}^{k^{*}} 2^{-2 k \varepsilon}\right)^{1 / 2} \leq c\|f\|_{H_{2}^{\alpha}} .
\end{aligned}
$$

The proof of Lemma 1 is complete. 
Local uniform convergence of the Riesz means of Laplace and Dirac expansions

\section{Sketch of the proofs of Lemmas 10 and 11}

The fundamental tool making possible these estimates is a new asymptotical formula for Bessel functions, given in [9]. The classical asymptotical expansions of the Bessel functions has the following form (see e.g. in Watson $[25])$ :

$$
\begin{aligned}
J_{\nu}(z)= & \sqrt{\frac{2}{z \pi}}\left(\cos \left(z-\frac{\pi \nu}{2}-\frac{\pi}{4}\right) \sum_{m=0}^{[(k-1) / 2]}(-1)^{m} \frac{(\nu, 2 m)}{(2 z)^{2 m}}+\right. \\
& \left.-\sin \left(z-\frac{\pi \nu}{2}-\frac{\pi}{4}\right) \sum_{m=0}^{[(k-2) / 2]}(-1)^{m} \frac{(\nu, 2 m+1)}{(2 z)^{2 m+1}}\right) \\
& +\mathrm{O}\left(\frac{\exp (\Im z)}{|z|^{k+1 / 2}}\right)
\end{aligned}
$$

if $z$ is not a negative real number nor zero. Here

$$
(\nu, m)=\frac{\Gamma(\nu+m+1 / 2)}{m ! \Gamma(\nu-m+1 / 2)}
$$

and the constant in the remainder term depends only on $\nu, k$ and $\delta$ if $\pi-|\arg (z)| \geq \delta>0$. To prove the lemmas we have to handle the quantities

$$
\frac{J_{\nu}(z)}{z^{\nu}}-\frac{J_{\nu}(\Re z)}{(\Re z)^{\nu}}, \quad \Re z \geq 1 \text {. }
$$

Substituting (32) we get the remainder term

$$
\underline{\underline{\mathrm{O}}}\left(\frac{\exp (|y|)}{|z|^{k+\nu+1 / 2}}\right), \quad x=\Re z, y=\Im z,
$$

which is not appropriate for our purposes. We have proved in [9] that (34) can be refined as

$$
\underline{\underline{\mathrm{o}}}\left(\frac{\exp (y)-1}{|z|^{k+\nu+1 / 2}}+\frac{|y|}{x^{k+\nu+3 / 2}}\right), \quad x \geq 1 .
$$

While estimating the integrals in Lemmas 10 and 11, we use the asymptotic expansion derived from (32) with remainder term (35) if $x$ is large and the 
Taylor series expansion of Bessel functions if $x$ is small. Throughout the proofs we distinguish the cases A) $\lambda \leq \rho^{n}$ and B) $\lambda \geq \rho_{n}$.

In case $\mathrm{A})$, we integrate by parts using the rules

$$
\begin{aligned}
\int r^{\nu+1} J_{\nu}(\mu r) \mathrm{d} r & =\frac{1}{\mu} r^{\nu+1} J_{\nu+1}(\mu r) \\
\int r^{-\nu} J_{\nu+1}(\mu r) \mathrm{d} r & =-\frac{1}{\mu} r^{-\nu} J_{\nu}(\mu r)
\end{aligned}
$$

in order to get a factor $\lambda / \rho_{n}$ in the expression. Then we split the integral into three parts:

$$
\int_{0}^{R}=\int_{0}^{R / \rho_{n}}+\int_{R / \rho_{n}}^{R / \lambda}+\int_{R / \lambda}^{R}
$$

and estimate separately using the asymptotic expansion (resp. Taylor series expansion) corresponding to the size of the argument of the Bessel function involved. The case B) is dealt with similarly but the partial integration is organized with opposite roles in order to get a factor $\rho_{n} / \lambda$.

Following these hints the (rather lengthy) proof of Lemmas 10 and 11 can be completed; the reader interested in more details is encouraged to contact the author.

Through Lemmas 10 and 11 the proof of Lemma 1 and then that of Theorems 1 and 2 became complete.

Remark. - We shall give here the construction of a Riesz basis $\left(u_{n}\right) \subset$ $L_{2}(\Omega)$ where $\Omega=(0, a)^{N}$ is an $N$-dimensional open cube, with eigenvalues

$$
\lambda_{n} \in \mathbb{C}, \quad \sup _{n}\left|\Im \lambda_{n}\right|=\infty .
$$

Take first the case $N=1$. Recall the following theorem of Vinogradov and Vasjunin [22, Part III]. Let $\left\{z_{k} \mid k \in \mathbb{Z}\right\}$ be a sequence satisfying the Carleson condition

$$
\inf _{n} \prod_{k \neq n} \frac{\left|z_{n}-z_{k}\right|}{\left|z_{n}-\bar{z}_{k}\right|}>0
$$

and suppose that

$$
\begin{array}{cl}
\Im z_{k}>0, & \lim _{k \rightarrow \infty} \Im z_{k}=+\infty . \\
& -690-
\end{array}
$$


Then for any $a>0$ the system $\left\{\exp \left(i z_{k} x\right) \mid k \in \mathbb{Z}\right\}$ can be completed to a system $\left\{\exp \left(i \mu_{n} x\right) \mid n \in \mathbb{Z}\right\}$ which is Riesz basis in $L_{2}(0, a)$ (after normalization). Now the system

$$
\left\{\exp \left(i\left[\mu_{n_{1}} x_{1}+\cdots+\mu_{n_{N}} x_{N}\right]\right) \mid n_{1}, \ldots, n_{N} \in \mathbb{Z}\right\}, \quad\left(x_{1}, \ldots, x_{N}\right) \in \Omega
$$

will be Riesz basis in $L_{2}(\Omega)$ with eigenvalue

$$
\lambda_{n}=\mu_{n_{1}}^{2}+\cdots+\mu_{n_{N}}^{2}, \quad n:=\left(n_{1}, \ldots, n_{N}\right) .
$$

In the case when $\mu_{n_{1}}=\cdots=\mu_{n_{N}}=z_{k}$, we get $\lambda_{n}=N z_{k}^{2}, \nu_{n}=N^{1 / 2} \Im z_{k}$ which tends to infinity if $k \rightarrow \infty$. Finally remark that (C) is equivalent to the conditions

$$
\inf _{\substack{n, k \\ n \neq k}} \frac{\left|z_{n}-z_{k}\right|}{\left|z_{n}-\bar{z}_{k}\right|}>0, \quad \sup _{n} \sum_{k} \Im z_{n} \Im z_{k}\left|z_{n}-\bar{z}_{k}\right|^{-2}<\infty .
$$

It is easy to check that e.g. the sequence

$$
z_{k}:=2^{|k|} \operatorname{sign} k+i 2^{|k| / 2}
$$

satisfies $\left(\mathrm{C}^{\prime}\right)$ and hence $(\mathrm{C})$.

\section{Dirac expansions}

In this final part of the paper, we consider the Dirac operator in $\mathbb{R}^{3}$, the relativistic variant of the Laplace operator in quantum mechanics. If the scalar and vector potentials vanish, the Dirac operator has the form

$$
H=h c \sum_{k=1}^{3} \gamma_{4} \gamma_{k} \frac{\partial}{\partial x_{k}}+\mu c^{2} \gamma_{4} .
$$

Hence $h$ is the Planck constant, $c$ is the velocity of light, $\mu$ is the rest mass of the particle and the Dirac matrices $\gamma_{1}, \ldots, \gamma_{4}$ are given by the Pauli matrices

$$
\begin{aligned}
\sigma_{1}=\left(\begin{array}{cc}
0 & -i \\
-i & 0
\end{array}\right) \quad \sigma_{2} & =\left(\begin{array}{cc}
0 & -1 \\
1 & 0
\end{array}\right) \quad \sigma_{3}=\left(\begin{array}{cc}
-i & 0 \\
0 & i
\end{array}\right) \\
& -691-
\end{aligned}
$$


by the rule

$$
\gamma_{4}=\left(\begin{array}{cc}
I & 0 \\
0 & -I
\end{array}\right) \quad \gamma_{k}=\left(\begin{array}{cc}
0 & \sigma_{k} \\
-\sigma_{k} & 0
\end{array}\right) \quad \text { and } \quad I=\left(\begin{array}{ll}
1 & 0 \\
0 & 1
\end{array}\right) .
$$

It is easy to check that

$$
\gamma_{4} \gamma_{k}=\left(\begin{array}{cc}
0 & \sigma_{k} \\
\sigma_{k} & 0
\end{array}\right)=-\gamma_{k} \gamma_{4}, \quad k=1,2,3 .
$$

Consider the eigenvalue problem

$$
H \underline{\psi}=\lambda \underline{\psi}, \quad \underline{\psi}=\left(\psi_{1}, \psi_{2}, \psi_{3}, \psi_{4}\right)^{T}
$$

where $\lambda \in \mathbb{C}$ is arbitrary, $\psi_{i}=\psi_{i}\left(x_{1}, x_{2}, x_{3}\right)$ for $1 \leq i \leq 4$. If we substitute (37) into (38), we get that

$$
H=\left(\begin{array}{cc}
\mu c^{2} I & A \\
A & -\mu c^{2} I
\end{array}\right)
$$

where

$$
A=h c \sum_{k=1}^{3} \sigma_{k} \frac{\partial}{\partial x_{k}}=h c\left(\begin{array}{cc}
-i \frac{\partial}{\partial x_{3}} & -i \frac{\partial}{\partial x_{1}}-\frac{\partial}{\partial x_{2}} \\
-i \frac{\partial}{\partial x_{1}}+\frac{\partial}{\partial x_{2}} & i \frac{\partial}{\partial x_{3}}
\end{array}\right) .
$$

Taking this into account, the eigenvalue problem takes the form

$$
\begin{aligned}
& A\left(\begin{array}{l}
\psi_{1} \\
\psi_{2}
\end{array}\right)=\left(\lambda+\mu c^{2}\right)\left(\begin{array}{l}
\psi_{3} \\
\psi_{4}
\end{array}\right) \\
& A\left(\begin{array}{l}
\psi_{3} \\
\psi_{4}
\end{array}\right)=\left(\lambda-\mu c^{2}\right)\left(\begin{array}{l}
\psi_{1} \\
\psi_{2}
\end{array}\right) .
\end{aligned}
$$

Lemma 12. - All the coordinates $\psi_{i}$ of the eigenfunctions (38) satisfy

$$
-\Delta \psi_{i}=\frac{\lambda^{2}-\mu^{2} c^{4}}{c^{2} h^{2}} \psi_{i}
$$

provided that $\underline{\psi}$ is twice continuously differentiable.

Proof. - We can easily check that

$$
\sigma_{i} \sigma_{j}= \begin{cases}\sigma_{k} & \text { if }(i, j, k) \text { is a cyclical permutation of }(1,2,3) \\ -\sigma_{k} & \text { otherwise. }\end{cases}
$$


Local uniform convergence of the Riesz means of Laplace and Dirac expansions

Then it is immediate from (40) that

$$
A^{2}=c^{2} h^{2}\left(\begin{array}{cc}
-\Delta & 0 \\
0 & -\Delta
\end{array}\right)
$$

Consequently (41) implies

$$
c^{2} h^{2}\left(\begin{array}{l}
-\Delta \psi_{1} \\
-\Delta \psi_{2}
\end{array}\right)=A^{2}\left(\begin{array}{l}
\psi_{1} \\
\psi_{2}
\end{array}\right)=\left(\lambda+\mu c^{2}\right) A\left(\begin{array}{l}
\psi_{3} \\
\psi_{4}
\end{array}\right)=\left(\lambda^{2}-\mu^{2} c^{4}\right)\left(\begin{array}{l}
\psi_{1} \\
\psi_{2}
\end{array}\right) .
$$

This proves (42) for $i=1,2$; the case $i=3,4$ is similar.

Now consider a bounded domain

$$
\Omega \subset \mathbb{R}^{3}
$$

and suppose that we are given a Riesz basis $\left(\underline{\psi}_{n}\right)_{n=1}^{\infty}$ in $L_{2}\left(\Omega, \mathbb{C}^{4}\right)$ of the eigenfunctions of the Dirac operator

$$
H \underline{\psi}_{n}=\lambda_{n} \underline{\psi}_{n} .
$$

Denote $\left(\underline{v}_{n}\right)_{1}^{\infty}$ the biorthogonal system, i.e., for which

$$
\left\langle\underline{v}_{n}, \underline{\psi}_{k}\right\rangle=\delta_{n, k} .
$$

Let $\mu_{n}$ be a square root of $\left(\lambda_{n}^{2}-\mu^{2} c^{4}\right) /\left(c^{2} h^{2}\right)$ with $\Re \mu_{n} \geq 0$ and let

$$
\rho_{n}:=\Re \mu_{n}, \quad \nu_{n}:=\Im \mu_{n} .
$$

Denote $\stackrel{\circ}{H}_{p}^{\alpha}\left(\Omega, \mathbb{C}^{4}\right)$ the space of all four-component vectors for which every component belongs to $\stackrel{\circ}{H}_{p}^{\alpha}(\Omega)$. Define the $\mu$-th Riesz means of order $s<1 / 2$ of $\underline{f}$ by

$$
\sigma_{\mu}^{s}(\underline{f}, x)=\sum_{\rho_{n}<\mu}\left\langle\underline{f}, \underline{v}_{n}\right\rangle \underline{\psi}_{n}(x)\left(1-\frac{\rho_{n}^{2}}{\lambda^{2}}\right)^{s} .
$$

In what follows the dimension $N$ always equals to 3 .

THEOREM 3.- Let $\underline{f} \in \stackrel{\circ}{H}_{p}^{\alpha}\left(\Omega, \mathbb{C}^{4}\right)$, supp $\underline{f} \subset \Omega, \alpha p>N, 1<p<\infty$, $0 \leq s<1 / 2, \alpha+s \geq(\bar{N}-1) / 2$. Then

$$
\sigma_{\lambda}^{s}(\underline{f}, x) \stackrel{\lambda \rightarrow \infty}{\longrightarrow} \underline{f}(x)
$$

locally uniformly in $x$. 
THEOREM 4.- Let $\underline{f} \in \stackrel{\circ}{H}_{2}^{\alpha}\left(\Omega, \mathbb{C}^{4}\right)$, supp $\underline{f} \subset \Omega, \alpha>0,0 \leq s<1 / 2$, $\alpha+s \geq(N-1) / 2$. Then

$$
\sigma_{\lambda}^{s}(\underline{f}, x) \stackrel{\lambda \rightarrow \infty}{\longrightarrow} 0, \quad x \in \Omega \backslash \operatorname{supp} f
$$

locally uniformly.

For the proof we remark first that the mean value formula (7) holds also with $\underline{\psi}_{n}$ instead of $u_{n}$ with

$$
\mu_{n}=\left(\lambda_{n}^{2}-\mu^{2} c^{4}\right)^{1 / 2}(h c)^{-1}
$$

this follows from (42). The other main tool is the square sum estimate (15). It has been proved in [6] and [9] under the assumption that the eigenfunctions $u_{n}$ form a Bessel system. Since $\left(\underline{\psi}_{n}\right)$ is Riesz basis, expanding $\underline{f}=(f, 0,0,0)^{T}$ we get that

$$
\sum\left|\left\langle f, \psi_{n, 1}\right\rangle\right|^{2}=\sum\left|\left\langle\underline{f}, \underline{\psi}_{n}\right\rangle\right|^{2} \leq c\|\underline{f}\|^{2}=c\|f\|^{2}
$$

so $\left(\psi_{n, 1}\right)$ is a Bessel system from eigenfunctions of the Laplace operator. The same is true for $\left(\psi_{n, k}\right), k=2,3,4$. Hence (15) holds with $\underline{\psi}_{n}$ instead of $u_{n}$. Since the whole proof for the Laplace operator is based on (7) and (15), it can be word by word repeated to obtain Theorems 3 and 4 . The only difference appears when proving Lemma 8 , we have to substitute $v^{\lambda}(r)$ by $\left(v^{\lambda}(r), 0,0,0\right)$ to obtain the desired equality in the first coordinate; other position of $v^{\lambda}(r)$ yields the equality in the other coordinates.

Remark. - An orthornormal basis from eigenfunctions of the Dirac operator is given in Evans [5], where the domain $\Omega$ is a sphere; in this system the least eigenvalue has infinite multiplicity.

\section{references}

[1] Alimov (S. A.) - - On spectral decompositions of functions in $H_{p}^{\alpha}$, Math. USSR Sbornik 30, $\mathrm{n}^{\circ} 1$ (1976), pp. 1-16.

[2] Alimov (S. A.) and Joó (I.) .- On the Riesz summability of eigenfunction expansions, Acta Sci. Math. Szeged 45 (1983), pp. 5-18. 
Local uniform convergence of the Riesz means of Laplace and Dirac expansions

[3] Bateman (H.) and ERdÉlyi (A.) .- Higher transcendental functions, McGrawHill, New York, 1-2 (1953).

[4] Bergh (J.) and Löfström (J.) .- Interpolation spaces, An introduction, Springer, Berlin (1976).

[5] Evans (W. D.) . - Eigenfunction expansions associated with the Dirac relativistic equations I-II, Quart. J. Math. Oxford 17 (1966), pp. 211-233; 18 (1967), pp. 239-262.

[6] HORVÁTH (M.) . - Sur le développement spectral de l'opérateur de Schrödinger, Comptes Rendus Acad. Sci. Paris, Série I, 311 (1990), pp. 499-502.

[7] HORVÁTH (M.) . - Exact norm estimates for the singular Schrödinger operator, Acta Math. Hung. 60 (1992), pp. 177-195.

[8] HORVÁTH (M.) .- Uniform estimations of the Green function for the singular Schrödinger operator, Acta Math. Hung. 61 (1993), pp. 327-342.

[9] HORVÁTH (M.) .- Local uniform convergence of the eigenfunction expansion associated with the Laplace operator I-II, Acta Math. Hung. 64 (1994), pp. 1-25, 101-138.

[10] HoRvÁTH (M.), Joó (I.) and KOMORNIK (V.) .- An equiconvergence theorem, Annales Univ. Sci. Budapest, Sectio Math. 31 (1988), pp. 19-26.

[11] IL'IN (V. A.) and Alimov (S. A.) .- Convergence conditions for the spectral expansions associated with selfadjoint extensions of elliptic operators I-II, Differencialnie Uravnenija (in Russian) 7, $\mathrm{n}^{\circ} 4$ (1971), pp. 670-710; 7, $\mathrm{n}^{\circ} 5$ (1971), pp. 851-882.

[12] Joó (I.) .- On the summability of eigenfunction expansions I, Acta Math. Hung. 43, $\mathrm{n}^{\circ}$ 1-2. (1984), pp. 73-83.

[13] Joó (I.) .- On the summability of eigenfunction expansions II, III, Annales Univ. Sci. Budapest, Sectio Math. 27 (1985), pp. 167-184; 28 (1986), pp. 253-262.

[14] JoÓ (I.) .- On the uniform Riesz summability of eigenfunction expansions I, Annales Univ. Sci. Budapest, Sectio Math. 31 (1988), pp. 191-211.

[15] Joó (I.) .- Exact estimate for the spectral function of the singular Schrödinger operator, Periodica Math. Hung. 18, $\mathrm{n}^{\circ} 3$ (1987), pp. 203-211.

[16] Joó (I.) .- On the divergence of eigenfunction expansions, Annales Univ. Sci. Budapest, Sectio Math. 32 (1989), pp. 3-36.

[17] Joó (I.) .- On the convergence of eigenfunction expansions, Acta Math. Hung. 60 (1992), pp. 125-156.

[18] JOÓ (I.) and KOMORNIK (V.) .- On the equiconvergence of expansions by Riesz bases formed by eigenfunctions of the Schrödinger operator, Acta Sci. Math. Szeged 46 (1983), pp. 357-375.

[19] KOMORNIK (V.) . - Sur l'équiconvergence des séries orthogonales et biorthogonales correspondant aux fonctions propres des opérateurs différentiels linéaires, Comptes Rendus Acad. Sci. Paris, Série I, 299 (1984), pp. 217-219.

[20] Nikiforov (A.) and Ouvarov (V.) .- Fonctions spéciales de la physique mathématique, Édition Mir, Moscou (1983).

[21] Nikolskil (S. M.) .- Approximation of functions of several variables and imbedding theorems, Nauka, Moscow (1977), in Russian. 


\section{Horváth}

[22] Nikolskil (N. K.), Pavlov (B. S.) and Hruscov (S. V.) .- Unconditional bases of exponentials and of reproducing kernels, Lecture Notes in Math., Springer, Berlin, 864 (1981), pp. 214-335.

[23] Titchmarsh (E. C.) .- Eigenfunction expansions associated with second order differential equations I-II, Clarendon Press, Oxford 1946, 1958.

[24] Triebel (H.) .- Interpolation theory. Functions spaces. Differential operators, V.E.B. Verlag, Berlin (1978).

[25] Watson (G. N.) . - A treatise on the theory of Bessel functions, Cambridge Univ. Press (1952).

[26] Young (R. M.) .- An introduction to nonharmonic Fourier series, Academic Press, New York (1980).

[27] ZYGmund (A.) .- Trigonometric Series, Cambridge Univ. Press (1959). 